\title{
A HIERARCHY OF MODELS FOR SUPERCONDUCTING THIN FILMS
}

\author{
S. J. CHAPMAN \\ D. R. HERON \\ MATHEMATICAL INSTITUTE, \\ 24-29 ST. GILES, \\ OXFORD OX1 3LB*
}

\begin{abstract}
A hierarchy of models for type-II superconducting thin films is presented. Through appropriate asymptotic limits this hierarchy passes from the mesoscopic Ginzburg-Landau model to the London model with isolated vortices as $\delta$ function singularities, to vortex-density models and finally to macroscopic critical-state models. At each stage it is found that a key nondimensional parameter is $\Lambda=\lambda^{2} / d L$ where $\lambda$ is the penetration depth of the magnetic field, a material parameter, and $d$ and $L$ are a typical thickness and lateral dimension of the film respectively. The models simplify greatly if this parameter is large or small.
\end{abstract}

Key words. superconductivity, vortices, thin films, homogenization, critical state.

AMS subject classifications. 82D 55

1. Introduction. The response of a bulk superconducting material to an applied magnetic field is conveniently described by Figure 1.1, which shows the phase the superconductor adopts as a function of the external magnetic field $H_{e x t}$ and the material parameter $\kappa$ (known as the Ginzburg-Landau parameter) which determines the type of superconducting material; $\kappa<1 / \sqrt{2}$ describes what are known as type-I superconductors, while $\kappa>1 / \sqrt{2}$ describes what are known as type-II superconductors.

For type-I superconductors in sufficiently low magnetic fields the material is in the superconducting state, and the field is excluded from the interior of the sample except in thin boundary layers (this effect is known as the Meissner effect). However, there is a critical magnetic field, $H_{c}$, above which the material will revert to the normally conducting (normal) state, and the magnetic field will penetrate it fully.

In type-II superconductors this critical magnetic field splits into a lower critical field, $H_{c_{1}}$ and an upper critical field $H_{c_{2}}$. For magnetic fields below $H_{c_{1}}$ the material is in the superconducting state and the field is excluded from the interior, while for magnetic fields above $H_{c_{2}}$ the material is in the normal state and the field penetrates it fully. For magnetic fields between $H_{c_{1}}$ and $H_{c_{2}}$ a third state exists, known as the "mixed state", in which there is a partial penetration of the magnetic field into the superconducting material, which occurs by means of thin filaments of non-superconducting material carrying magnetic flux ("flux tubes") and circled by a vortex of superconducting current (hence these filaments are often referred to as vortices).

A hierarchy of models for bulk type-II superconductors has been derived recently in [6]. The starting point for this hierarchy is the Ginzburg-Landau (GL) model, which applies on mesoscopic lengthscales $\sim 0.01 \mu \mathrm{m}$, and which is generally believed to describe the behaviour of superconductors well (at least for low-temperature superconductors not too far from the critical temperature $T_{c}$.) The GL model is quite complicated, and through the asymptotic limit $\kappa \rightarrow \infty$ may be simplified to the London model, a linear equation in which the vortices appear as line singularities. These may then be averaged to produce vortexdensity models. Finally, if vortex pinning by inhomogeneities is included in these vortex-density models, so called critical-state models can be derived.

Here we are interested in the simplifications which arise when the superconducting material comprises a thin film, possibly of varying thickness. Such a situation is very common experimentally and technologically because of the relative ease with which thin films can be manufactured. We will derive a hierarchy of models for superconducting thin films corresponding exactly to those for bulk superconductors. We will find that a key nondimensional parameter is $\Lambda=\lambda_{\text {eff }} / L$, where $L$ is a typical lateral dimension of the film and the effective screening length [24] $\lambda_{\text {eff }}=\lambda^{2} / d$, where $\lambda$ is the penetration depth of the magnetic field, a material parameter, and $d$ is a typical thickness of the film. The canonical scaling is for $\Lambda$ to be of order one as $d / L \rightarrow 0$, in which case the problems for the electric current in the film and the magnetic field outside it are coupled. The problem simplifies greatly if $\Lambda$ is either large or small, since in each case the problems for the electric current and the magnetic field decouple. Many of the models we arrive at are new; our aim is to show where the existing models fit into the general framework, and to fill in the gaps. In particular we

*tel: +44 1865 270506, fax: +44 1865 270515, email: chapman@maths.ox.ac.uk 


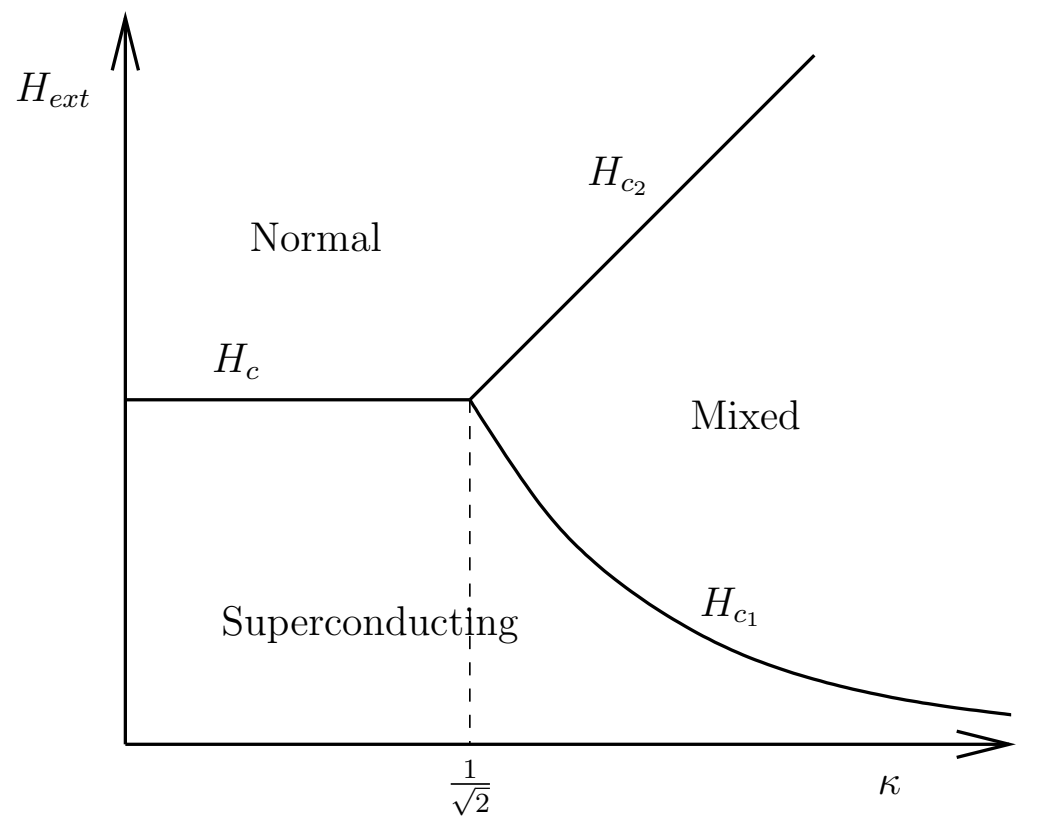

FIG. 1.1. The response of a superconducting material as a function of the applied magnetic field $H_{\text {ext }}$ and the GinzburgLandau parameter $\kappa$.

will find that the thin-film limit of the Ginzburg-Landau model considered in [8] corresponds to the limit $\Lambda \rightarrow \infty$, while the thin-film critical-state models studied recently in [29] and [31] correspond to the limit $\Lambda \rightarrow 0$.

Before we begin let us first make a note of some of the lengthscales in the problem. There are two material parameters which are lengthscales, both of which appear in the Ginzburg-Landau model. These are $\lambda$, the aforementioned penetration depth, which is the typical lengthscale for the decay of magnetic field away from a vortex, and $\xi$, the coherence length, which is the typical lengthscale for the variation in the number density of superconducting electrons, and is the vortex core radius. The ratio of these two lengthscales is the Ginzburg-Landau parameter $\kappa=\lambda / \xi$; as we have already said, for type-II supercoductors $\kappa>1 / \sqrt{2}$, and the London model corresponds to the limit in which $\kappa \rightarrow \infty$. However, we will find that the important parameter in determining the behaviour of thin films is not $\kappa$ but $\kappa_{\text {eff }}=\lambda_{\text {eff }} / \xi=(\lambda / d) \kappa$. Thus when $d$ is small compared to $\lambda$ the Ginzburg-Landau parameter is enhanced. The situation is not disimilar to that of lubrication theory, in which the key parameter is the reduced Reynolds number rather than the Reynolds number itself.

To go with these two material lengths we have three geometrical lengths in the problem. Since we will allow the film to vary in thickness, besides the typical thickness $d$ and lateral dimension $L$ of the film we also have the typical lengthscale for the thickness variations, which we will denote by $\delta$ (we assume that the amplitude of the thickness variations is of the same order as the thickness itself, which is the canonical case). When pinning through inhomogeneities is introduced we have the typical lengthscale for variation of the pinning potential, which we denote by $\varepsilon$. Finally, we have the typical separation of vortices, which we denote by $\nu$, and which will be determined by the strength of the applied magetic field.

Thus there are seven lengthscales in the problem, and it is the relative sizes of these which will determine which is the relevant thin film model in any given situation.

In Section 2 we introduce the Ginzburg-Landau theory which underpins our hierarchy of models, and consider its thin film limit. In Section 3 we consider the London limit $\kappa_{\text {eff }} \rightarrow \infty$ of the thin-film GinzburgLandau model, and show that this is the same as the thin-film limit of the bulk London model. In Section 4 we let the spearation of vortices $\nu / L \rightarrow 0$ and average the thin-film London model to produce a thin-film vortex-density model. We show that this is the same as the thin-film limit of the bulk vortex-density model.

In Section 5 we let the lengthscale of the pinning potential $\varepsilon \rightarrow 0$ and homogenise the pinning force to produce a thin-film critical-state model. Finally, in Section 6, we present our conclusions. 
2. Ginzburg-Landau models. The starting point for our discussion of thin-film models of superconductivity is the Ginzburg-Landau equations. In their steady form these equations were written down by Ginzburg and Landau in [17], through the minimisation of a phenomenologically-developed free-energy functional. Later it was shown by Gor'kov [18] that they could be derived as a limit of the microscopic Bardeen, Cooper \& Schrieffer (BCS) model [2]. Time-dependent versions of the Ginzburg-Landau equations were written down by Schmidt [27], and in 1968 Gor'kov and Eliashberg [19] demonstrated that the following equations (2.1)-(2.2) could be derived from the BCS model for a superconductor with paramagnetic impurities. These correspond to a gauge-invariant gradient flow of the Ginzburg-Landau energy functional, and as such are the simplest time-dependent equations whose solutions evolve to the minimisers of that functional. The dimensionless time-dependent Ginzburg-Landau equations for a superconducting material occupying a region $\Omega \subseteq \mathbb{R}^{3}$ are

$$
\begin{aligned}
\frac{1}{\kappa^{2}} \frac{\partial \Psi}{\partial t}+\frac{i \Psi \phi}{\kappa} & =\left(\frac{1}{\kappa} \nabla-i \boldsymbol{A}\right)^{2} \Psi+\Psi\left(1-|\Psi|^{2}\right) \text { in } \Omega, \\
-(\operatorname{curl})^{2} \boldsymbol{A}-\frac{\sigma}{\kappa^{2}}\left(\frac{\partial \boldsymbol{A}}{\partial t}+\nabla \phi\right) & =\frac{i}{2 \kappa}\left(\Psi^{*} \nabla \Psi-\Psi \nabla \Psi^{*}\right)+|\Psi|^{2} \boldsymbol{A} \text { in } \Omega,
\end{aligned}
$$

where $\Psi$ is the normalised superconducting order parameter, so that $|\Psi|^{2}$ represents the number density of superconducting electrons, with $|\Psi|=1$ representing wholly superconducting material and $\Psi=0$ representing wholly nonsuperconducting (normal) material; $\boldsymbol{A}$ and $\phi$ are the magnetic vector potential and electric scalar potential respectively, which are such that the magnetic field $\boldsymbol{H}$ and electric field $\boldsymbol{E}$ are given by

$$
\begin{aligned}
\boldsymbol{H} & =\operatorname{curl} \boldsymbol{A}, \\
\boldsymbol{E} & =-\frac{\partial \boldsymbol{A}}{\partial t}-\nabla \phi .
\end{aligned}
$$

$\boldsymbol{A}$ is determined up to a gradient; once $\boldsymbol{A}$ is given $\phi$ is determined up to a function of $t$. The constant $\sigma$ is a measure of the normal conductivity of the superconducting material (in this nondimensional form $\sigma$ is the ratio of the timescale for diffusion of magnetic field in the normal state to the timescale for the relaxation of the order parameter), and $\kappa$ is the Ginzburg-Landau parameter. Length has been scaled with the penetration depth $\lambda$, which is the natural lengthscale for variations in the magnetic field. In these units the thermodynamic critical field is given by $H_{c}=1 / \sqrt{2}$, and the upper and lower critical fields are given by $H_{c_{2}}=\kappa$ and $H_{c_{1}} \sim \log (\kappa) / 2 \kappa$ for large $\kappa$ respectively.

The most common boundary conditions on (2.1)-(2.2) are the natural conditions

$$
\boldsymbol{n} \cdot(\nabla \Psi-i \boldsymbol{A} \Psi)=0 \quad \text { on } \partial \Omega,
$$

and continuity of $\boldsymbol{A}, \boldsymbol{H}$ and $\boldsymbol{E}$ across $\partial \Omega$ (assuming that the permeability and permittivity of the region exterior to the superconductor is equal to that of the superconducting material; the modification if this is not the case is easy to make). Equation (2.5) is applicable when the region adjacent to the superconductor is an insulator, which is the case we will consider henceforth. Note that (2.5) implies that no supercurrent passes through the boundary, and we assume also that no normal current is applied directly to the superconductor.

Outside $\Omega$ we have Maxwell's equations (neglecting displacement current)

$$
\begin{aligned}
\operatorname{curl} \boldsymbol{H} & =\boldsymbol{J}_{e x t}, \\
\operatorname{div} \boldsymbol{H} & =0, \\
\boldsymbol{H}_{t}+\operatorname{curl} \boldsymbol{E} & =\mathbf{0}, \\
\operatorname{div} \boldsymbol{E} & =0,
\end{aligned}
$$

where $\boldsymbol{J}_{\text {ext }}$ is the externally imposed current which is driving the system. The most commonly considered situation is that in which a uniform magnetic field is applied at infinity, in which case $\boldsymbol{J}_{\text {ext }}$ is zero and

$$
\boldsymbol{H} \rightarrow \boldsymbol{H}_{\text {ext }} \text { as }|\boldsymbol{x}| \rightarrow \infty
$$

(assuming a bounded superconducting region). The continuity of $\boldsymbol{A}, \boldsymbol{H}$ and $\boldsymbol{E}$ are the usual boundary conditions on Maxwell's equations at an interface between two media. Note though that these conditions 
are not all independent, since for example the continuity of the normal component of $\boldsymbol{H}$ arises by taking (2.7) to hold in a generalised sense everywhere, but this equation is automatic from (2.3), which, if it holds in a generalised sense, gives continuity of the tangential components of $\boldsymbol{A}$.

Equations (2.1)-(2.2) are gauge invariant in the sense that they are invariant under transformations of the form

$$
\boldsymbol{A} \rightarrow \boldsymbol{A}+\nabla \omega, \quad \phi \rightarrow \phi-\frac{\partial \omega}{\partial t}, \quad \Psi \rightarrow \Psi e^{i \kappa \omega}
$$

We make take advantage of this invariance to write the equations in terms of real variables by writing

$$
\Psi=f e^{i \chi}, \quad \boldsymbol{Q}=\boldsymbol{A}-\frac{1}{\kappa} \nabla \chi, \quad \Phi=\phi+\frac{1}{\kappa} \frac{\partial \chi}{\partial t},
$$

to give

$$
\begin{aligned}
-\frac{1}{\kappa^{2}} \frac{\partial f}{\partial t}+\frac{1}{\kappa^{2}} \nabla^{2} f & =f^{3}-f+f|\boldsymbol{Q}|^{2} \quad \text { in } \Omega, \\
f^{2} \Phi+\operatorname{div}\left(f^{2} \boldsymbol{Q}\right) & =0 \quad \text { in } \Omega, \\
-(\operatorname{curl})^{2} \boldsymbol{Q} & =\frac{\sigma}{\kappa^{2}}\left(\frac{\partial \boldsymbol{Q}}{\partial t}+\nabla \Phi\right)+f^{2} \boldsymbol{Q} \quad \text { in } \Omega, \\
\boldsymbol{H} & =\operatorname{curl} \boldsymbol{Q}, \\
\boldsymbol{E} & =-\frac{\partial \boldsymbol{Q}}{\partial t}-\nabla \Phi .
\end{aligned}
$$

The vortex solutions characteristic of type-II superconductors can be illustrated by seeking a solution of the form

$$
\begin{array}{r}
\Psi=f(r) e^{i n \theta}, \\
\boldsymbol{A}=A(r) \boldsymbol{e}_{\theta},
\end{array}
$$

on an infinite domain, where $n$ is an integer known as the vortex number, $r$ and $\theta$ are polar coordinates, and $\boldsymbol{e}_{\theta}$ is the unit vector in the azimuthal direction. Substituting into (2.1)-(2.2) gives

$$
\begin{aligned}
& \frac{1}{\kappa^{2}} \frac{1}{r} \frac{d}{d r}\left(r \frac{d f}{d r}\right)-\left(A-\frac{n}{\kappa r}\right)^{2} f=f^{3}-f, \\
& \frac{d}{d r}\left(\frac{1}{r} \frac{d}{d r}(r A)\right)=f^{2}\left(A-\frac{n}{\kappa r}\right), \\
& f, \quad A \text { bounded as } r \rightarrow 0, \\
& f \rightarrow 1, \quad A \rightarrow 0 \text { as } r \rightarrow \infty .
\end{aligned}
$$

The existence of a solution which necessarily has $f(0)=0$ has been shown by Berger and Chen [3]. The supercurrent is given by

$$
\boldsymbol{J}=-f^{2}\left(A-\frac{n}{\kappa r}\right) \boldsymbol{e}_{\theta},
$$

which shows the vortex nature of this solution. The axial magnetic field carried by the vortex is

$$
\int_{\mathbb{R}^{2}} \boldsymbol{H} \cdot \boldsymbol{d} \boldsymbol{S}=\frac{2 \pi n}{\kappa}
$$

which is quantized in units of $2 \pi / \kappa$, with $n$ the number of flux quanta carried by the vortex. Note that for large values of $\kappa, f \approx 1$ except in a region of order $\kappa^{-1}$ from the origin, which is the vortex core.

Since the flux quantum is $O(1 / \kappa)$ it is common to rescale magnetic field with $1 / \kappa$ when considering vortex solutions. However, when considering the thin-film limit we must first rescale length so that we are working on the lateral dimension of the sample, $L$ say, which requires a corresponding rescaling of time with 
$L^{2} / \lambda^{2}$. The canonical scale for the magnetic field then involves rescaling with $\lambda^{2} /\left(L^{2} \kappa\right)$ (if we assume that the vortex separation is $O(L)$; see [10] for example), so that $\boldsymbol{Q}$ and $\boldsymbol{J}$ must be rescaled with $\lambda /(L \kappa)$. This gives

$$
\begin{aligned}
\frac{\xi^{2}}{L^{2}}\left(-\frac{\partial f}{\partial t}+\nabla^{2} f\right) & =f^{3}-f+\frac{\xi^{2} f|\boldsymbol{Q}|^{2}}{L^{2}} \text { in } \Omega, \\
f^{2} \Phi+\operatorname{div}\left(f^{2} \boldsymbol{Q}\right) & =0 \text { in } \Omega, \\
-\lambda^{2} / L^{2} \operatorname{curl} \boldsymbol{H} & =-\boldsymbol{J}=\frac{\xi^{2} \sigma}{L^{2}}\left(\frac{\partial \boldsymbol{Q}}{\partial t}+\nabla \Phi\right)+f^{2} \boldsymbol{Q} \text { in } \Omega, \\
\boldsymbol{H} & =\operatorname{curl} \boldsymbol{Q},
\end{aligned}
$$

remembering that $\kappa=\lambda / \xi$. Note also the equations

$$
\begin{aligned}
\operatorname{div} \boldsymbol{H} & =0, \\
\operatorname{div} \boldsymbol{J} & =0,
\end{aligned}
$$

which follow from (2.27) and (2.28) and will prove useful in the thin-film analysis. With these equations we have the boundary conditions

$$
\begin{aligned}
\frac{\partial f}{\partial n} & =0 \text { on } \partial \Omega, \\
\boldsymbol{Q} \cdot \boldsymbol{n} & =0 \text { on } \partial \Omega, \\
\boldsymbol{J} \cdot \boldsymbol{n} & =0 \text { on } \partial \Omega,
\end{aligned}
$$

with $\boldsymbol{H}$ and $\boldsymbol{E}$ continuous across $\partial \Omega$.

2.1. Vortex Pinning in the Ginzburg-Landau model. Before we go on to consider the thin-film version of (2.25)-(2.33) let us first describe the modifications which can be made to model the pinning of vortices in this framework.

In most technological applications superconductors are required to carry a transport current. The interaction of this current with the current circling a vortex causes the vortex to move (this is often considered to be the result of the "Lorentz force" on the magnetic flux line carried by the vortex due to the transport current). The motion of the vortex dissipates energy, leads to an electric field, and hence a nonzero resistivity, and is therefore undesirable. In practice attempts are made to "pin" vortices at certain sites in the material in order to impede their motion. It is found that any form of inhomogeneity (for example impurities, dislocations or grain boundaries) will help to pin vortices. Such impurities have the effect of impeding locally the ability of the material to become superconducting. A popular way of modelling this inhomogeneity in the GinzburgLandau framework is to allow the equilibrium density of superconducting electrons to vary spatially $[23,7]$. In the simplest case this leads to

$$
\begin{aligned}
\frac{\xi^{2}}{L^{2}}\left(-\frac{\partial f}{\partial t}+\nabla^{2} f\right) & =f^{3}-a(\boldsymbol{x}) f+\frac{\xi^{2} f|\boldsymbol{Q}|^{2}}{L^{2}} \text { in } \Omega, \\
f^{2} \Phi+\operatorname{div}\left(f^{2} \boldsymbol{Q}\right) & =0 \text { in } \Omega, \\
-\lambda^{2} / L^{2} \operatorname{curl} \boldsymbol{H} & =-\boldsymbol{J}=\frac{\xi^{2} \sigma}{L^{2}}\left(\frac{\partial \boldsymbol{Q}}{\partial t}+\nabla \Phi\right)+f^{2} \boldsymbol{Q} \text { in } \Omega,
\end{aligned}
$$

where the equilibrium density of superconducting electrons is denoted by $a(\boldsymbol{x})$. Of course, more generally we may allow the coefficient of $|\Psi|^{2} \Psi$ as well as $\lambda, \xi, \kappa$ and $\sigma$ to vary spatially, but we will consider here the case when these parameters are constant.

Numerical simulations of these equations in two dimensions show that the vortices are attracted to minima of $a$, as we would have hoped [7, 14].

2.2. Thin-film limit of the Ginzburg-Landau model. Let the film be given, here and throughout, by

$$
\Omega=\{(x, y, z):(x, y) \in D,(\zeta-g / 2) \epsilon<z<(\zeta+g / 2) \epsilon\},
$$


where $D \subset \mathbb{R}^{2}$ is the projection of the film in the $(x, y)$ plane, $\zeta(x, y)$ is the height of the centresurface of the film, $g(x, y)$ is the film thickness, and $\epsilon=d / L$ is the aspect ratio.

We write $\xi / L=\Xi$ and $\lambda^{2} / L^{2}=\epsilon \Lambda$, since the canonical scaling will turn out to be $\Lambda=O(1)$. Then

$$
\begin{aligned}
\Xi^{2}\left(-\frac{\partial f}{\partial t}+\nabla^{2} f\right) & =f^{3}-a(\boldsymbol{x}) f+\Xi^{2} f|\boldsymbol{Q}|^{2} \text { in } \Omega, \\
f^{2} \Phi+\operatorname{div}\left(f^{2} \boldsymbol{Q}\right) & =0 \text { in } \Omega, \\
-\epsilon \Lambda \operatorname{curl} \boldsymbol{H} & =-\boldsymbol{J}=\Xi^{2} \sigma\left(\frac{\partial \boldsymbol{Q}}{\partial t}+\nabla \Phi\right)+f^{2} \boldsymbol{Q} \text { in } \Omega,
\end{aligned}
$$

We consider first the problem in the film, where we rescale $z$ with $\epsilon$ by setting $z=\epsilon Z$. Henceforth for clarity we use $\boldsymbol{h}$ to denote the magnetic field outside the film and $\boldsymbol{H}$ to denote it inside the film. Then

$$
\begin{aligned}
& \Xi^{2}\left(-\frac{\partial f}{\partial t}+\frac{\partial^{2} f}{\partial x^{2}}+\frac{\partial^{2} f}{\partial y^{2}}+\frac{1}{\epsilon^{2}} \frac{\partial^{2} f}{\partial Z^{2}}\right)=f^{3}-f+\Xi^{2} f\left(Q_{1}^{2}+Q_{2}^{2}+Q_{3}^{2}\right) \text { in } \Omega, \\
& f^{2} \Phi+\frac{\partial\left(f^{2} Q_{1}\right)}{\partial x}+\frac{\partial\left(f^{2} Q_{2}\right)}{\partial y}+\frac{1}{\epsilon} \frac{\partial\left(f^{2} Q_{3}\right)}{\partial Z}=0 \text { in } \Omega, \\
& -\epsilon \Lambda\left(\frac{\partial H_{3}}{\partial y}-\frac{1}{\epsilon} \frac{\partial H_{2}}{\partial Z}\right)=-J_{1}=\Xi^{2} \sigma\left(\frac{\partial Q_{1}}{\partial t}+\frac{\partial \Phi}{\partial x}\right)+f^{2} Q_{1} \text { in } \Omega, \\
& -\epsilon \Lambda\left(\frac{1}{\epsilon} \frac{\partial H_{1}}{\partial Z}-\frac{\partial H_{3}}{\partial x}\right)=-J_{2}=\Xi^{2} \sigma\left(\frac{\partial Q_{2}}{\partial t}+\frac{\partial \Phi}{\partial y}\right)+f^{2} Q_{2} \text { in } \Omega, \\
& -\epsilon \Lambda\left(\frac{\partial H_{2}}{\partial x}-\frac{\partial H_{1}}{\partial y}\right)=-J_{3}=\Xi^{2} \sigma\left(\frac{\partial Q_{3}}{\partial t}+\frac{1}{\epsilon} \frac{\partial \Phi}{\partial Z}\right)+f^{2} Q_{3} \text { in } \Omega, \\
& \frac{\partial Q_{3}}{\partial y}-\frac{1}{\epsilon} \frac{\partial Q_{2}}{\partial Z}=H_{1} \\
& \frac{1}{\epsilon} \frac{\partial Q_{1}}{\partial Z}-\frac{\partial Q_{3}}{\partial x}=H_{2} \\
& \frac{\partial Q_{2}}{\partial x}-\frac{\partial Q_{1}}{\partial y}=H_{3} \\
& \frac{\partial H_{1}}{\partial x}+\frac{\partial H_{2}}{\partial y}+\frac{1}{\epsilon} \frac{\partial H_{3}}{\partial Z}=0 \\
& \frac{\partial J_{1}}{\partial x}+\frac{\partial J_{2}}{\partial y}+\frac{1}{\epsilon} \frac{\partial J_{3}}{\partial Z}=0 .
\end{aligned}
$$

With these equations we have the boundary conditions on the upper and lower surfaces of the film

$$
\begin{gathered}
-\epsilon^{2} \frac{\partial}{\partial x}(\zeta \pm g / 2) \frac{\partial f}{\partial x}(x, y, \zeta \pm g / 2)-\epsilon^{2} \frac{\partial}{\partial y}(\zeta \pm g / 2) \frac{\partial f}{\partial y}(x, y, \zeta \pm g / 2) \\
+\frac{\partial f}{\partial Z}(x, y, \zeta \pm g / 2)=0 \text { for }(x, y) \in D \\
-\epsilon \frac{\partial}{\partial x}(\zeta \pm g / 2) Q_{1}(x, y, \zeta \pm g / 2)-\epsilon \frac{\partial}{\partial y}(\zeta \pm g / 2) Q_{2}(x, y, \zeta \pm g / 2) \\
+Q_{3}(x, y, \zeta \pm g / 2)=0 \text { for }(x, y) \in D \\
-\epsilon \frac{\partial}{\partial x}(\zeta \pm g / 2) J_{1}(x, y, \zeta \pm g / 2)-\epsilon \frac{\partial}{\partial y}(\zeta \pm g / 2) J_{2}(x, y, \zeta \pm g / 2) \\
+J_{3}(x, y, \zeta \pm g / 2)=0 \text { for }(x, y) \in D \\
\boldsymbol{h}(x, y, z=\epsilon(\zeta \pm g / 2))=\boldsymbol{H}(x, y, Z=\zeta \pm g / 2)
\end{gathered}
$$

We formally expand all variables in a power series in $\epsilon$ as $\epsilon \rightarrow 0$ as

$$
\boldsymbol{H}=\boldsymbol{H}^{(0)}+\epsilon \boldsymbol{H}^{(1)}+\cdots,
$$


etc. Then at leading order in (2.41)-(2.50)

$$
\begin{aligned}
\frac{\partial^{2} f^{(0)}}{\partial Z^{2}} & =0 \text { in } \Omega, \\
\frac{\partial\left(\left(f^{(0)}\right)^{2} Q_{3}^{(0)}\right)}{\partial Z} & =0 \text { in } \Omega, \\
\Lambda \frac{\partial H_{2}^{(0)}}{\partial Z} & =-J_{1}^{(0)}=\Xi^{2} \sigma\left(\frac{\partial Q_{1}^{(0)}}{\partial t}+\frac{\partial \Phi^{(0)}}{\partial x}\right)+\left(f^{(0)}\right)^{2} Q_{1}^{(0)} \text { in } \Omega, \\
-\Lambda \frac{\partial H_{1}^{(0)}}{\partial Z} & =-J_{2}^{(0)}=\Xi^{2} \sigma\left(\frac{\partial Q_{2}^{(0)}}{\partial t}+\frac{\partial \Phi^{(0)}}{\partial y}\right)+\left(f^{(0)}\right)^{2} Q_{2}^{(0)} \text { in } \Omega, \\
0 & =\frac{\partial \Phi^{(0)}}{\partial Z} \text { in } \Omega, \\
\frac{\partial Q_{2}^{(0)}}{\partial Z} & =0, \\
\frac{\partial Q_{1}^{(0)}}{\partial Z} & =0, \\
\frac{\partial Q_{2}^{(0)}}{\partial x}-\frac{\partial Q_{1}^{(0)}}{\partial y} & =H_{3}^{(0)}, \\
\frac{\partial H_{3}^{(0)}}{\partial Z} & =0, \\
\frac{\partial J_{3}^{(0)}}{\partial Z} & =0,
\end{aligned}
$$

with boundary conditions

$$
\begin{aligned}
\frac{\partial f^{(0)}}{\partial Z}(x, y, \zeta \pm g / 2) & =0 \text { for }(x, y) \in D, \\
Q_{3}^{(0)}(x, y, \zeta \pm g / 2) & =0 \text { for }(x, y) \in D, \\
J_{3}^{(0)}(x, y, \zeta \pm g / 2) & =0 \text { for }(x, y) \in D, \\
\boldsymbol{h}^{(0)}(x, y, z= \pm 0) & =\boldsymbol{H}^{(0)}(x, y, Z=\zeta \pm g / 2) .
\end{aligned}
$$

Equations (2.55) and (2.65) give $f^{(0)}=f^{(0)}(x, y, t)$. From (2.64) and (2.67) we see that $J_{3}^{(0)}=0$. We see from (2.59)-(2.61) that $Q_{1}^{(0)}, Q_{2}^{(0)}$ and $\Phi^{(0)}$ are independent of $Z$, so that $J_{1}^{(0)}$ and $J_{2}^{(0)}$ are independent of $Z$. Then (2.57) and (2.58) may be integrated to give

$$
\begin{aligned}
& H_{1}^{(0)}=\frac{Z J_{2}^{(0)}}{\Lambda}+a(x, y, t), \\
& H_{2}^{(0)}=-\frac{Z J_{1}^{(0)}}{\Lambda}+b(x, y, t) .
\end{aligned}
$$

Similarly (2.63) gives

$$
H_{3}^{(0)}=c(x, y, t) .
$$

Now evaluating on the top and bottom of the film $Z=\zeta \pm g / 2$ and using (2.68) gives

$$
\left[\boldsymbol{h}^{(0)}\right]=\frac{g}{\Lambda}\left(J_{2}^{(0)},-J_{1}^{(0)}, 0\right) \quad \text { for } \quad(x, y) \in D,
$$

where the square bracket indicates the jump in the quantity enclosed across $D$. Writing this jump condition in the more usual form gives

$$
\left[\boldsymbol{e}_{z} \wedge \boldsymbol{h}^{(0)}\right]=\frac{g}{\Lambda} \boldsymbol{J}_{7}^{(0)} \quad \text { for } \quad(x, y) \in D
$$




$$
\left[\boldsymbol{e}_{z} \cdot \boldsymbol{h}^{(0)}\right]=0 \quad \text { for } \quad(x, y) \in D
$$

where $\boldsymbol{e}_{z}$ is the unit vector in the $z$-direction. Equations (2.73)-(2.74) form boundary conditions on the problem for the external magnetic field, which satisfies the Maxwell equations

$$
\begin{aligned}
\operatorname{curl} \boldsymbol{h}^{(0)} & =\boldsymbol{J}_{\text {ext }}^{(0)}, \\
\operatorname{div} \boldsymbol{h}^{(0)} & =0, \\
\boldsymbol{h}^{(0)} & \rightarrow \boldsymbol{h}_{\text {ext }}^{(0)} \quad \text { as }|\boldsymbol{r}| \rightarrow \infty .
\end{aligned}
$$

As we might expect, $\boldsymbol{h}^{(0)}$ is simply the magnetic field generated if the total current in the film were distributed on a sheet, plus the applied (externally generated) magnetic field. We can therefore calculate $\boldsymbol{h}^{(0)}$ once we have found $\boldsymbol{J}^{(0)}$.

Returning to the film, we see from (2.56) and (2.66) that $Q_{3}^{(0)}=0$. What remains is to find an equation for $f^{(0)}, Q_{1}^{(0)}, Q_{2}^{(0)}$ and $\Phi^{(0)}$. To do this we need to proceed to higher orders in the expansion of (2.41) and $(2.51)$

At first order in (2.41) and (2.51) we find

$$
\frac{\partial f^{(1)}}{\partial Z}=0
$$

At second order we find

$(2.79) \Xi^{2}\left(-\frac{\partial f^{(0)}}{\partial t}+\frac{\partial^{2} f^{(0)}}{\partial x^{2}}+\frac{\partial^{2} f^{(0)}}{\partial y^{2}}+\frac{\partial^{2} f^{(2)}}{\partial Z^{2}}\right)=\left(f^{(0)}\right)^{3}-a(\boldsymbol{x}) f^{(0)}+\Xi^{2} f^{(0)}\left(\left(Q_{1}^{(0)}\right)^{2}+\left(Q_{2}^{(0)}\right)^{2}\right)$ in $\Omega$,

with

$$
\begin{aligned}
-\frac{\partial}{\partial x}(\zeta \pm g / 2) \frac{\partial f^{(0)}}{\partial x}(x, y, \zeta \pm g / 2)-\frac{\partial}{\partial y}(\zeta \pm g / 2) & \frac{\partial f^{(0)}}{\partial y}(x, y, \zeta \pm g / 2) \\
& +\frac{\partial f^{(2)}}{\partial Z}(x, y, \zeta \pm g / 2)=0 \text { for }(x, y) \in D
\end{aligned}
$$

Integrating (2.79) from $\zeta-g / 2$ to $\zeta+g / 2$ and using (2.80) gives

$$
\Xi^{2}\left(-\frac{\partial f^{(0)}}{\partial t}+\frac{1}{g} \nabla \cdot\left(g \nabla f^{(0)}\right)\right)=\left(f^{(0)}\right)^{3}-f^{(0)}+\Xi^{2} f^{(0)}\left(\left(Q_{1}^{(0)}\right)^{2}+\left(Q_{2}^{(0)}\right)^{2}\right) \text { for }(x, y) \in D
$$

We are still missing an equation for the divergence of $\boldsymbol{Q}^{(0)}$, which should come from equation (2.42). Proceeding to next order in this equation gives

$$
\left(f^{(0)}\right)^{2} \Phi^{(0)}+\frac{\partial\left(\left(f^{(0)}\right)^{2} Q_{1}^{(0)}\right)}{\partial x}+\frac{\partial\left(\left(f^{(0)}\right)^{2} Q_{2}^{(0)}\right)}{\partial y}+\frac{\partial\left(\left(f^{(0)}\right)^{2} Q_{3}^{(1)}\right)}{\partial Z}=0 \text { in } \Omega .
$$

At next order in the boundary condition (2.52) we find

$$
\begin{aligned}
-\frac{\partial}{\partial x}(\zeta \pm g / 2) Q_{1}^{(0)}(x, y, \zeta \pm g / 2)-\frac{\partial}{\partial y}(\zeta \pm g / 2) Q_{2}^{(0)}(x, y, \zeta \pm g / 2) & \\
& +Q_{3}^{(1)}(x, y, \zeta \pm g / 2)=0 \text { for }(x, y) \in D .
\end{aligned}
$$

Now, integrating (2.82) from $Z=\zeta-g / 2$ to $Z=\zeta+g / 2$ and using (2.83) gives

$$
\left(f^{(0)}\right)^{2} \Phi^{(0)}+\frac{1}{g} \nabla \cdot\left(g\left(f^{(0)}\right)^{2} \boldsymbol{Q}^{(0)}\right)=0 \text { for }(x, y) \in D .
$$

A similar analysis on (2.50) and (2.53) gives

$$
\nabla \cdot\left(\begin{array}{c}
g \boldsymbol{J}^{(0)} \\
8
\end{array}\right)=0
$$


Finally, by continuity of magnetic field at the interface (2.68) and constancy of $H_{3}$ in $Z$ (2.71), equation (2.62) becomes

$$
\frac{\partial Q_{2}^{(0)}}{\partial x}-\frac{\partial Q_{1}^{(0)}}{\partial y}=h_{3}^{(0)}(x, y, 0) .
$$

Summary. We now have a closed model for the leading-order problem as $\epsilon \rightarrow 0$ with $\Xi$ and $\Lambda$ fixed. Dropping the superscripts for clarity we have

$$
\begin{aligned}
\Xi^{2}\left(-\frac{\partial f}{\partial t}+\frac{1}{g} \nabla \cdot(g \nabla f)\right) & =f^{3}-a(\boldsymbol{x}) f+\Xi^{2} f|\boldsymbol{Q}|^{2} \text { for }(x, y) \in D, \\
f^{2} \Phi+\frac{1}{g} \nabla \cdot\left(g f^{2} \boldsymbol{Q}\right) & =0 \text { for }(x, y) \in D, \\
\frac{\partial Q_{2}}{\partial x}-\frac{\partial Q_{1}}{\partial y} & =h_{3}(x, y, 0) \quad \text { for } \quad(x, y) \in D, \\
Q_{3} & =0, \\
\nabla \cdot(g \boldsymbol{J}) & =0, \\
\boldsymbol{J} & =-\Xi^{2} \sigma\left(\frac{\partial \boldsymbol{Q}}{\partial t}+\nabla \Phi\right)-f^{2} \boldsymbol{Q} \quad \text { for } \quad(x, y) \in D, \\
J_{3} & =0, \\
{\left[\boldsymbol{e}_{z} \wedge \boldsymbol{h}\right] } & =\frac{g}{\Lambda} \boldsymbol{J} \quad \text { for } \quad(x, y) \in D, \\
{\left[\boldsymbol{e}_{z} \cdot \boldsymbol{h}\right] } & =0 \text { for } \quad(x, y) \in D, \\
\operatorname{curl} \boldsymbol{h} & =\boldsymbol{J}_{\text {ext }}, \\
\operatorname{div} \boldsymbol{h} & =0, \\
\boldsymbol{h} & \rightarrow \boldsymbol{h}_{\text {ext }} \text { as }|\boldsymbol{x}| \rightarrow \infty .
\end{aligned}
$$

If $g$ is constant then (2.87)-(2.93) are simply the two-dimensional Ginzburg-Landau equations. The big difference in the thin film case is that $h_{3}$ is not related to $\boldsymbol{J}$ through $\boldsymbol{J}=$ curl $\left(0,0, h_{3}\right)$, but through the exterior problem (2.94)-(2.98).

We note that there is an equivalent complex formulation

$$
\begin{aligned}
\Xi^{2}\left(-\frac{\partial \Psi}{\partial t}-i \Psi \phi+\frac{1}{g}(\nabla-i \boldsymbol{A}) \cdot g(\nabla-i \boldsymbol{A}) \Psi\right) & =\Psi\left(|\Psi|^{2}-a(\boldsymbol{x})\right) \text { for }(x, y) \in D, \\
\frac{\partial A_{2}}{\partial x}-\frac{\partial A_{1}}{\partial y} & =h_{3}(x, y, 0) \quad \text { for } \quad(x, y) \in D, \\
A_{3} & =0, \\
{\left[\boldsymbol{e}_{z} \wedge \boldsymbol{h}\right] } & =\frac{g}{\Lambda} \boldsymbol{J} \quad \text { for } \quad(x, y) \in D, \\
{\left[\boldsymbol{e}_{z} \cdot \boldsymbol{h}\right] } & =0 \text { for } \quad(x, y) \in D, \\
\nabla \cdot(g \boldsymbol{J}) & =0, \\
-\Xi^{2} \sigma\left(\frac{\partial \boldsymbol{A}}{\partial t}+\nabla \phi\right)+\frac{1}{2}\left(\Psi^{*} \nabla \Psi-\Psi \nabla \Psi^{*}\right)-|\Psi|^{2} \boldsymbol{A} & =\boldsymbol{J} \quad \text { for } \quad(x, y) \in D, \\
J_{3} & =0, \\
\operatorname{curl} \boldsymbol{h} & =\boldsymbol{J}_{\text {ext }}, \\
\operatorname{div} \boldsymbol{h} & =0, \\
\boldsymbol{h} & \rightarrow \boldsymbol{h}_{\text {ext }} \text { as }|\boldsymbol{x}| \rightarrow \infty,
\end{aligned}
$$

where

$$
\Psi=f e^{i \chi}, \quad \boldsymbol{Q}=\underset{9}{\boldsymbol{A}-\nabla \chi, \quad \Phi=\phi+\frac{\partial \chi}{\partial t},}
$$


and $\chi$ is arbitrary. To these equations we must add the boundary conditions (2.5) (or equivalently (2.31)$(2.32)$ ) and (2.33) on the lateral edges of the film, giving

$$
\begin{aligned}
\boldsymbol{\nu} \cdot(\nabla \Psi-i \boldsymbol{A} \Psi) & =0 \quad \text { on } \partial D, \\
\boldsymbol{J} \cdot \boldsymbol{\nu} & =0 \quad \text { on } \partial D,
\end{aligned}
$$

where $\boldsymbol{\nu}$ is the unit outward normal to $\partial D$.

Before proceeding let us first examine the flow of information in the equations, and in particular whether we have the right number of equations and unknowns. As we have already mentioned, equations (2.102)(2.103) and (2.107)-(2.109) form a standard problem in magnetostatics, namely to determine the magnetic field generated by a surface current sheet. Thus these equations determine $\boldsymbol{h}$ if $\boldsymbol{J}$ is given. Having determined $\boldsymbol{h}$ equations (2.100)-(2.101) then determine $\boldsymbol{A}$ up to a gradient, which we can fix due to the gauge invariance property (2.110). With $\boldsymbol{A}$ given equations (2.99) and (2.104) (using (2.105)) with the boundary conditions (2.111)-(2.112) form a closed system for $\Psi$ and $\phi$. Finally, the loop is closed by (2.105), which gives $\boldsymbol{J}$ as a function of $\Psi, \phi$ and $\boldsymbol{A}$.

2.2.1. Thin strip geometry. Suppose our thin film is a thin strip of width $2 l$, i.e. $D=(x, y):-l<x<l$, and that $\boldsymbol{J}_{\text {ext }}$ and $\boldsymbol{h}_{\text {ext }}$ are such that $\boldsymbol{h}=\left(h_{1}, 0, h_{3}\right), \boldsymbol{J}=\left(0, J_{2}, 0\right)$, with $h_{1}, h_{3}$ and $J_{2}$ independent of $y$. Then we can write the relationship between $h_{3}$ and $J_{2}$ explicitly using a Hilbert transform as follows. We first write $\boldsymbol{h}=\boldsymbol{h}_{a p p}+\boldsymbol{h}^{\prime}$, where $\boldsymbol{h}_{a p p}$ is the total applied magnetic field (generated by $\boldsymbol{J}_{\text {ext }}$ and $\boldsymbol{h}_{e x t}$ ), so that $\boldsymbol{h}^{\prime}$ satisfies the homogeneous version of (2.107) and tends to zero at infinity. Then equations (2.107) and (2.108) imply that we can write

$$
\begin{aligned}
\boldsymbol{h}^{\prime} & =\nabla w, \\
\nabla^{2} w & =0,
\end{aligned}
$$

where $\nabla$ is the two-dimensional gradient in $x$ and $z$, with

$$
\begin{aligned}
{\left[\frac{\partial w}{\partial x}\right] } & =\frac{g J_{2}}{\Lambda}, \\
{\left[\frac{\partial w}{\partial z}\right] } & =0, \\
w & \rightarrow 0 \quad \text { as } x^{2}+z^{2} \rightarrow \infty .
\end{aligned}
$$

Since the solution to (2.114)-(2.117) will be symmetric, so that

$$
w(x, z)=-w(x,-z)
$$

we may consider the problem in the upper half-plane only, giving

$$
\begin{aligned}
\nabla^{2} w & =0, \quad \text { in } z>0, \\
\frac{\partial w}{\partial x} & =\frac{g J_{2}}{2 \Lambda}, \quad \text { on } z=0, \\
\frac{\partial w}{\partial z} & =h_{3}(x, 0)-h_{a p p, 3}(x, 0), \quad \text { on } z=0, \\
w & \rightarrow 0, \quad \text { as } x^{2}+z^{2} \rightarrow \infty .
\end{aligned}
$$

Then

$$
\left.\frac{\partial w}{\partial z}\right|_{z=0}=\mathcal{H}\left(\left.\frac{\partial w}{\partial x}\right|_{z=0}\right) \equiv \frac{1}{\pi} f_{-\infty}^{\infty} \frac{1}{\bar{x}-x} \frac{\partial w}{\partial x}(\bar{x}, 0) d \bar{x},
$$

so that

$$
h_{3}(x, 0)-h_{a p p, 3}(x, 0)=\frac{1}{\pi} \int_{-l}^{l} \frac{g J_{2}(\bar{x})}{2 \Lambda(\bar{x}-x)} d \bar{x},
$$

This equation replaces (2.102)-(2.103) and (2.107)-(2.109) in (2.99)-(2.112). 


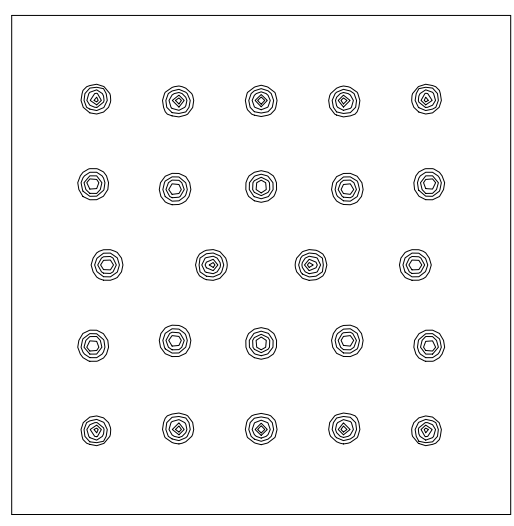

(a) Constant thickness film.

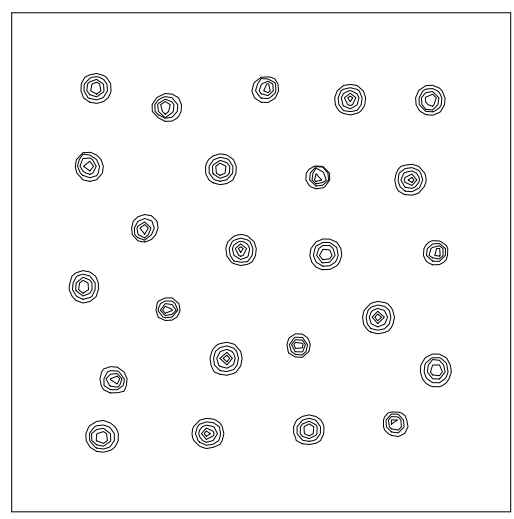

(c) Variable thickness film

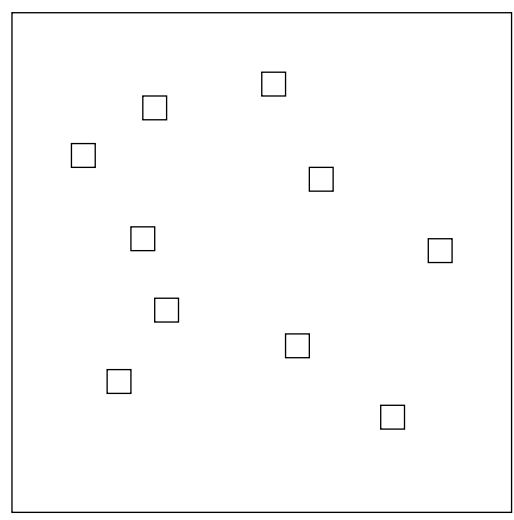

(b) Distribution of thin regions.

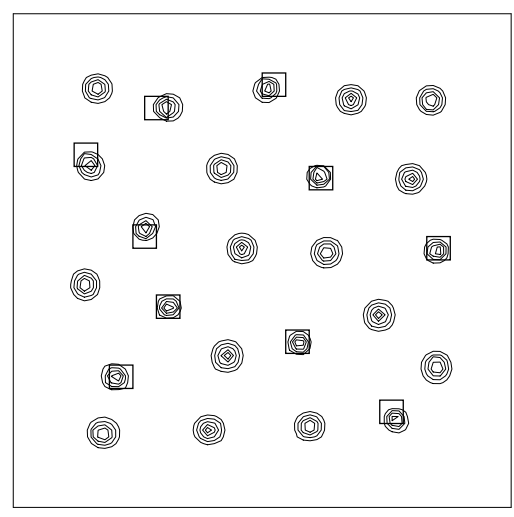

(d) Figs. 2.1(b) and 2.1(c) superimposed

FIG. 2.1. Level curves of the magnitude of the order parameter for superconducting samples having sides equal to 20 coherence lengths. Reproduced from [8].

2.2.2. The limit $\Lambda \rightarrow \infty$ with $\Xi$ fixed. There are three key parameters left in the model, namely $\Lambda$, $\Xi$ and $\sigma$. We have so far considered the canonical scaling in which all the equations remain coupled in the thin-film limit. The thin-film limit of the Ginzburg-Landau equations considered in [8] corresponds to the limit $\Lambda \rightarrow \infty$ with $\Xi$ fixed, since they consider the limit $\epsilon \rightarrow 0$ with fixed $\lambda, \xi, L$ and $\kappa$. As $\Lambda \rightarrow \infty$ we see from (2.94) that the current in the film is insufficient to affect the magnetic field at leading order, and the problems for $\boldsymbol{A}$ and $\Psi$ decouple leading to a great simplification. At leading order the applied magnetic field simply passes straight through the film, so that

$$
\boldsymbol{h}=\boldsymbol{h}_{\text {app }}, \quad \boldsymbol{A}=\left(0, x H_{3, a p p}, 0\right),
$$

where $\boldsymbol{h}_{a p p}$ is the total applied magnetic field (that generated by the external current plus that imposed at infinity), which is the solution to (2.107)-(2.109). Equations (2.99) and (2.104) can then be solved for $\Psi$ and $\phi$ since $\boldsymbol{A}$ is known. Note that the factor $\Xi^{2}$ may be removed from these equations by a rescaling of $\boldsymbol{x}$ and $\boldsymbol{A}$, as is done in [8]. The current in the film is then determined from (2.105). The correction to the magnetic field can then be calculated from (2.102)-(2.103) and (2.107)-(2.109). Thus in this expansion for large $\Lambda$ at each stage we have to solve the standard magnetostatics problem of determining the magnetic field due to a known surface current sheet, and then a problem for $\Psi$ and $\phi$ in which $\boldsymbol{A}$ is known.

The numerical solutions in [8] show that vortices are attracted to the minima of $g(x)$, that is, the 
thin parts of the film. Figure 2.1 shows a typical calculation, performed on a square film with side of (nondimensional) length $20(L=\xi$ is chosen as the unit of length, so dimensionally the side of the square is $20 \xi$ ), with applied field perpendicular to the film and of (nondimensional) strength 0.5. In (a) the solution for a film of constant thickness is shown (contours show the level curves of the modulus of the order parameter). Ten squares of size $\xi \times \xi$ were then chosen randomly, and the thickness of the film on the squares reduced to half that of the remaining film; the distribution of the thin regions is shown in (b). Figure 2.1(c) shows the position of the vortices for the variable thickness film with the same magnetic field strength as (a). Figure (d) shows figures (b) and (c) superimposed, so that the position of the vortices can be compared to the position of the thin regions of the film.

Numerical solutions of the time dependent verion of this reduced model are also performed in [1] to study vortex nucleation at boundaries in the presense of applied magnetic fields and currents.

The limit $\Lambda \rightarrow 0$ with $\Xi$ fixed. The alternative limit is $\Lambda \rightarrow 0$ with $\Xi$ fixed. In this case, from equations (2.94) and (2.92), we must rescale $\boldsymbol{J}, \boldsymbol{Q}$ and $\Phi$ with $\Lambda$. Then to leading order equation (2.89) gives

$$
h_{3}(x, y, 0)=0 \text { for }(x, y) \in D \text {. }
$$

Equations (2.96)-(2.98) can then be solved with this boundary condition for $\boldsymbol{h}$.

Equation (2.125) is valid away from vortices. Near each vortex an inner problem must be solved in which length is rescaled with $\Lambda$, which couples the problems for $\boldsymbol{h}$ and $\boldsymbol{J}$ together again. The limit $\Lambda \rightarrow 0$ will be much more interesting when we go on to consider vortex density models in Section 4 .

\section{London Models.}

3.1. The London limit of the thin-film Ginzburg-Landau model. From (2.87) we see that the vortex core radius is of order $\Xi$ in these units. The London model, corresponding to vanishing core radius, therefore corresponds to the limit $\Xi \rightarrow 0$. Since we are taking this limit after having let $\epsilon \rightarrow 0$ we are in the parameter regime $\epsilon \ll \Xi \ll 1$. We will consider the alternative regime $\Xi \ll \epsilon \ll 1$ in Section 3.3.

In the limit $\Xi \rightarrow 0$ with $\Lambda$ fixed we see that equation (2.87) implies $f^{2}=a(\boldsymbol{x})$ except at vortices. Then from (2.92) we have

$$
\boldsymbol{J}=-a \boldsymbol{Q}
$$

so that (2.89) gives

$$
\boldsymbol{e}_{z} \cdot \operatorname{curl}\left(\frac{\boldsymbol{J}}{a}\right)+h_{3}(x, y, 0)=0
$$

except at vortices.

Suppose there is a vortex at the origin. Near the vortex we rescale $r=\left(x^{2}+y^{2}\right)^{1 / 2}=\Xi R$, and expand

$$
\begin{aligned}
f & =f^{(0)}+\cdots, \\
\boldsymbol{Q} & =\frac{1}{\Xi} \boldsymbol{Q}^{(0)}+\cdots .
\end{aligned}
$$

Then at leading order in the core region

$$
\begin{aligned}
\boldsymbol{Q}^{(0)} & =-\frac{1}{R} \boldsymbol{e}_{\theta} \\
\frac{\partial^{2} f^{(0)}}{\partial R^{2}}+\frac{1}{R} \frac{\partial f^{(0)}}{\partial R}-\frac{f^{(0)}}{R^{2}} & =\left(f^{(0)}\right)^{3}-a(0) f^{(0)} .
\end{aligned}
$$

Writing

as in $[12]$ we find $\bar{f}$ satisfies

$$
f^{(0)}=\sqrt{a(0)} \bar{f}(\rho), \quad \rho=\sqrt{a(0)} R,
$$

$$
\begin{aligned}
\bar{f}^{\prime \prime}+\frac{\bar{f}^{\prime}}{\rho}-\frac{\bar{f}}{\rho^{2}} & =\bar{f}^{3}-\bar{f}, \\
\bar{f}(0) & =0, \\
\bar{f} & \rightarrow 1 \quad \text { as } \rho \rightarrow \infty,
\end{aligned}
$$


where ${ }^{\prime} \equiv d / d \rho$. Hence $\bar{f}$ is simply the solution corresponding to a two-dimensional rectilinear isotropic vortex, whose existence and uniqueness has been shown in [13]. From (3.5) and (3.1) we see the matching condition on the outer solution $\boldsymbol{J}$ as a vortex is approached is therefore

$$
\boldsymbol{J} \sim \frac{a(0)}{r} \boldsymbol{e}_{\theta} \text { as } r \rightarrow 0 .
$$

We may combined this matching condition succinctly with the London equation (3.2) by writing

$$
\boldsymbol{e}_{z} \cdot \operatorname{curl}\left(\frac{\boldsymbol{J}}{a(\boldsymbol{x})}\right)+h_{3}(x, y, 0)=2 \pi \delta(\boldsymbol{x}),
$$

where $\boldsymbol{x}=(x, y)$ and $\delta(\boldsymbol{x})$ is the two-dimensional Dirac $\delta$-function. Thus we have reduced the nonlinear but regular Ginzburg-Landau equation to a linear but singular London equation. The great advantage of the linearity of (3.10) is that if we have many vortices located at the positions $\boldsymbol{x}_{n}, n=0, \ldots, N$ we may simply add up their contributions to the electric current and magnetic field by the principle of superposition, to give

$$
\boldsymbol{e}_{z} \cdot \operatorname{curl}\left(\frac{\boldsymbol{J}}{a(\boldsymbol{x})}\right)+h_{3}(x, y, 0)=2 \pi \sum_{n=0}^{N} \delta\left(\boldsymbol{x}-\boldsymbol{x}_{n}\right) .
$$

All that remains is to determine a law of motion for each vortex, so that we can determine the evolution of the positions $\boldsymbol{x}_{n}$. To do this we need to proceed further down the asymptotic expansions. The analysis parallels exactly that in $[11,12,10]$. Asymptotically expanding the solution of (3.11), (2.91) in inner coordinates as $r \rightarrow 0$ as in [10] we find

$$
\boldsymbol{Q}=-\frac{1}{a} \boldsymbol{J} \sim-\frac{1}{\Xi R} \boldsymbol{e}_{\theta}-\frac{|\log \Xi|}{2(a g)}\left(\begin{array}{c}
(a g)_{y} \\
-(a g)_{x}
\end{array}\right)+\cdots,
$$

where the second term is evaluated at $r=0$ (and is therefore constant). Thus the expansion in the inner region must proceed as

$$
\begin{aligned}
f & =f^{(0)}+\Xi|\log \Xi| f^{(1)}+\cdots, \\
\boldsymbol{Q} & =\frac{1}{\Xi} \boldsymbol{Q}^{(0)}+|\log \Xi| \boldsymbol{Q}^{(1)}+\cdots, \\
\Phi & =\frac{|\log \Xi|}{\Xi} \Phi^{(0)}+\cdots, \\
\boldsymbol{v} & =|\log \Xi| \boldsymbol{v}^{(0)}+\cdots,
\end{aligned}
$$

where $\boldsymbol{v}$ is the velocity of the vortex at the origin. Equating coefficients of $|\log \Xi|$ in (2.87), (2.88), (2.91) and $(2.92)$ we find

$$
\begin{aligned}
& \boldsymbol{v}^{(0)} \cdot \boldsymbol{e}_{r} \frac{d f^{(0)}}{d R}+\frac{\partial^{2} f^{(1)}}{\partial R^{2}}+\frac{1}{R} \frac{\partial f^{(1)}}{\partial R}-\frac{f^{(1)}}{R^{2}}+\frac{1}{R^{2}} \frac{\partial^{2} f^{(1)}}{\partial \theta^{2}} \\
& (3.18)\left(f^{(0)}\right)^{2} \Phi^{(0)}-\frac{1}{R} \frac{\partial}{\partial \theta}\left(\frac{2 f^{(0)} f^{(1)}}{R}\right)+\frac{1}{R} \frac{\partial}{\partial R}\left(R\left(f^{(0)}\right)^{2} \boldsymbol{Q}^{(1)} \cdot \boldsymbol{e}_{r}\right)+\frac{1}{R} \frac{\partial}{\partial \theta}\left(\left(f^{(0)}\right)^{2} \boldsymbol{Q}^{(1)} \cdot \boldsymbol{e}_{\theta}\right)=0, \\
& \frac{1}{R} \frac{\partial}{\partial R}\left(R \boldsymbol{Q}^{(1)} \cdot \boldsymbol{e}_{\theta}\right)-\frac{1}{R} \frac{\partial}{\partial \theta}\left(\boldsymbol{Q}^{(1)} \cdot \boldsymbol{e}_{r}\right)=0, \\
& -\frac{1}{R} \frac{\partial}{\partial \theta}\left(\frac{2 f^{(0)} f^{(1)}}{R}\right)+\frac{1}{R} \frac{\partial}{\partial R}\left(R\left(f^{(0)}\right)^{2} \boldsymbol{Q}^{(0)} \cdot \boldsymbol{e}_{r}\right)+\frac{1}{R} \frac{\partial}{\partial \theta}\left(R\left(f^{(0)}\right)^{2} \boldsymbol{Q}^{(0)} \cdot \boldsymbol{e}_{\theta}\right) \\
& +\sigma\left(\frac{1}{R} \frac{\partial}{\partial R}\left(R \frac{\partial \Theta^{(0)}}{\partial R}\right)+\frac{1}{R^{2}} \frac{\partial^{2} \Theta^{(0)}}{\partial \theta^{2}}\right)=0
\end{aligned}
$$


From (3.18) and (3.20) we see that

$$
\left(f^{(0)}\right)^{2} \Phi^{(0)}=\sigma\left(\frac{1}{R} \frac{\partial}{\partial R}\left(R \frac{\partial \Theta^{(0)}}{\partial R}\right)+\frac{1}{R^{2}} \frac{\partial^{2} \Theta^{(0)}}{\partial \theta^{2}}\right) .
$$

As $R \rightarrow 0$ we have the boundary condition [11]

$$
\Phi^{(0)} \sim-\frac{1}{R} \boldsymbol{v}^{(0)} \cdot \boldsymbol{e}_{\theta} .
$$

From (3.19) we may write $\boldsymbol{Q}^{(1)}$ in terms of a scalar potential $\psi$ as

$$
\begin{aligned}
\boldsymbol{Q}^{(1)} \cdot \boldsymbol{e}_{\theta} & =\frac{1}{R} \frac{\partial \psi}{\partial \theta}, \\
\boldsymbol{Q}^{(1)} \cdot \boldsymbol{e}_{r} & =\frac{\partial \psi}{\partial R} .
\end{aligned}
$$

To match with the outer behaviour (3.12) we require

$$
\psi \sim \text { constant } \times R \sin (\theta-\alpha),
$$

for some constant angle $\alpha$. Thus we make the ansatz [12]

$$
\begin{aligned}
\psi & =a(0)^{-1 / 2} \phi(\rho) \sin (\theta-\alpha), \\
\Phi^{(0)} & =U a(0)^{1 / 2} \eta(\rho) \sin (\theta-\alpha), \\
f^{(1)} & =F(\rho) \cos (\theta-\alpha), \\
\boldsymbol{v}^{(0)} & =U\left(\cos (\theta-\alpha) \boldsymbol{e}_{r}-\sin (\theta-\alpha) \boldsymbol{e}_{\theta}\right)=U\left(\cos \alpha \boldsymbol{e}_{x}+\sin \alpha \boldsymbol{e}_{y}\right) .
\end{aligned}
$$

The system (3.17), (3.20), (3.21) then reduces to

$$
\begin{array}{r}
\frac{1}{\rho}\left(\rho F^{\prime}\right)^{\prime}-3 \bar{f}^{2} F+F=-U \bar{f}^{\prime}-\frac{2 \bar{f} \phi}{\rho^{2}}+\frac{2 F}{\rho^{2}}, \\
\frac{1}{\rho}\left(\rho \bar{f}^{2} \phi^{\prime}\right)^{\prime}+\sigma U\left(\eta^{\prime}+\frac{\eta}{\rho}\right)^{\prime}-\frac{\bar{f}^{2} \phi}{\rho^{2}}+\frac{2 \bar{f} F}{\rho^{2}}=0, \\
\eta^{\prime \prime}+\frac{1}{\rho} \eta^{\prime}-\frac{1}{\rho^{2}} \eta-\frac{f^{(0)^{2}} \eta}{\sigma}=0,
\end{array}
$$

where $^{\prime} \equiv d / d \rho$. Equation (3.22) gives the boundary condition

$$
\eta \sim \frac{1}{\rho} \quad \text { as } \rho \rightarrow 0
$$

on equation (3.31). Matching with the outer solution gives the second condition:

$$
\rho \eta \rightarrow 0 \quad \text { as } \rho \rightarrow \infty .
$$

This gives a well-posed problem for $\eta$, and numerical solutions of (3.31) with boundary conditions (3.32) and (3.33) have been given by Peres and Rubinstein [25].

We consider (3.29) and (3.30). Noting that the derivative of the leading-order solution $\bar{f}$ satisfies the homogeneous version of equation (3.29) we see that there will be a solution if and only if a certain solvability condition is satisfied. To derive this condition we multiply (3.29) by $\rho \bar{f}^{\prime}$, the derivative of (3.6) by $\rho f^{(1)}$ and subtract to obtain

$$
\rho U\left(\bar{f}^{\prime}\right)^{2}+\left(\rho F^{\prime} \bar{f}^{\prime}-\rho F \bar{f}^{\prime \prime}\right)^{\prime}=-\frac{2 \bar{f}^{\prime} \bar{f} \phi}{\rho}+\frac{2 \bar{f} F}{\rho^{2}} .
$$


Using (3.30) to eliminate the final $F$, integrating over $(0, \infty)$ and using the asymptotic behaviour of the $\bar{f}$ and $\Theta^{(0)}$ at 0 and $\infty$ we find

$$
\lim _{\rho \rightarrow \infty}\left(\phi^{\prime}+\frac{\phi}{\rho}\right)=-U \beta
$$

where

$$
\beta=\int_{0}^{\infty} \rho\left(\bar{f}^{\prime}\right)^{2} d \rho+\int_{0}^{\infty} \bar{f}^{2} \eta d \rho .
$$

Hence

$$
\lim _{\rho \rightarrow \infty} \boldsymbol{Q}^{(1)}=\frac{U \beta}{2}\left(\sin \alpha \boldsymbol{e}_{x}-\cos \alpha \boldsymbol{e}_{y}\right) .
$$

Matching with the outer solution (3.12) using (3.28) gives

$$
\boldsymbol{v}^{(0)}=-\frac{1}{\beta} \nabla \log (a g),
$$

so that to leading order

$$
\boldsymbol{v}=-\frac{|\log \Xi|}{\beta} \nabla \log (a g) .
$$

We see that vortices are attracted to the minima of $a g$. If we proceed to higher orders we find that the first two terms in the vortex velocity law give

$$
\boldsymbol{v}=-\frac{|\log \Xi|}{\beta} \nabla \log (a g)+\frac{2}{\beta a} \boldsymbol{J} \wedge \boldsymbol{e}_{z},
$$

where $\boldsymbol{J}$ is the regular part of the current density. The second term here becomes dominant either when $a g$ is constant, so that the first term vanishes, or when the background current density $\boldsymbol{J}$ is large, which will be the case when we allow the vortex separation $\nu / L$ to tend to zero to arrive at vortex-density models in Section $4^{1}$.

Summary. The leading-order London limit of the thin-film Ginzburg-Landau model is (dropping the superscripts for clarity)

$$
\begin{aligned}
\operatorname{curl} \boldsymbol{h} & =\boldsymbol{J}_{\text {ext }}, \\
\operatorname{div} \boldsymbol{h} & =0,
\end{aligned}
$$

with

$$
\begin{aligned}
{\left[\boldsymbol{e}_{z} \wedge \boldsymbol{h}\right] } & =\frac{g}{\Lambda} \boldsymbol{J} \quad \text { for } \quad(x, y) \in \Omega, \\
{\left[\boldsymbol{e}_{z} \cdot \boldsymbol{h}\right] } & =0, \\
\boldsymbol{h}^{(0)} & \rightarrow \boldsymbol{h}_{\text {ext }} \quad \text { as } \quad|\boldsymbol{r}| \rightarrow \infty
\end{aligned}
$$

for the magnetic field outside the superconductor, and

$$
\begin{aligned}
\boldsymbol{e}_{z} \cdot \operatorname{curl}\left(\frac{\boldsymbol{J}}{a}\right)+h_{3}(x, y, 0) & =2 \pi \sum_{n} \delta\left(\boldsymbol{x}-\boldsymbol{x}_{n}\right), \\
\operatorname{div}(g \boldsymbol{J}) & =0,
\end{aligned}
$$

\footnotetext{
${ }^{1}$ Strictly speaking the law of motion needs to be rederived when the terms in (3.40) switch order so that the second one dominates, but the analysis proceeds in exactly the same way and (3.40) still holds.
} 
with

$$
\boldsymbol{J} \cdot \boldsymbol{\nu}=0 \text { on } \partial D
$$

for the electric current inside the superconductor, with the vortices moving according to the law

$$
\dot{\boldsymbol{x}}_{n}=-\frac{|\log \Xi|}{\beta} \nabla \log (a g)+\frac{2}{\beta a} \boldsymbol{J} \wedge \boldsymbol{e}_{z} .
$$

The key equation here is (3.46); as we have already said, all the other equations are simply the thin-film versions of Maxwells equations. We note that there are two source terms in equation (3.46) for the current. The first is the sum of $\delta$-functions due to the vortices, as expected. The second is due to the applied magnetic field, which acts as a negative distributed vorticity. This term is due to the Meissner effect, by which a superconductor attempts to exclude a magnetic field from its interior. The current generated by this term in (3.46) is an attempt to shield the superconductor from the applied field, and will result in a lower magnetic field inside the superconductor than the applied field $\boldsymbol{h}_{e x t}$.

Note that if we instead consider vortices with negative winding number (corresponding to a magnetic field in the $-\boldsymbol{e}_{z}$ direction rather than the $\boldsymbol{e}_{z}$ direction), then (3.46) and (3.49) are modified to

$$
\begin{aligned}
\boldsymbol{e}_{z} \cdot \operatorname{curl}\left(\frac{\boldsymbol{J}}{a}\right)+h_{3}(x, y, 0) & =-2 \pi \sum_{n} \delta\left(\boldsymbol{x}-\boldsymbol{x}_{n}\right), \\
\dot{\boldsymbol{x}}_{n} & =-\frac{|\log \Xi|}{\beta} \nabla \log (a g)-\frac{2}{\beta a} \boldsymbol{J} \wedge \boldsymbol{e}_{z} .
\end{aligned}
$$

Note also that by writing the equations in terms of the total current in the film $g \boldsymbol{J}$ (rather than the average current $\boldsymbol{J}$ ) the solution depends only on the product ag. This shows how variations in film thickness play exactly the same role as variations in the equilibrium density of superconducting electrons.

These equations were derived in $[10,21]$ for the case $a=1$, and in [16] for the case of a strip geometry $D=(x, y):-l<x<l$ in the absence of vortices, with $a=g=1$. In the strip case (3.41)-(3.45) may be replaced by

$$
h_{3}(x, 0)-h_{a p p, 3}(x, 0)=\frac{1}{\pi} \int_{-l}^{l} \frac{g J_{2}(\bar{x})}{2 \Lambda(\bar{x}-x)} d \bar{x},
$$

as in Section 2.2.1.

The increased Ginzburg-Landau parameter. Note that from (2.94) the lengthscale for the decay of the magnetic field away from a vortex is $\Lambda$, which is the nondimensional version of Pearl's effective screening length for thin films [24]. Thus the London limit we have considered is valid so long as $\Xi \ll \Lambda$. Thus in a thin film the relevant parameter in determining the type of superconductor is not the Ginzburg-Landau parameter $\kappa$, but the increased Ginzburg-Landau parameter

$$
\kappa_{\mathrm{eff}}=\frac{\Lambda}{\Xi}=\frac{\lambda_{\mathrm{eff}}}{\xi}=\frac{\lambda^{2}}{\xi d}=\frac{\lambda \kappa}{d}
$$

This explains why thin films of even type-I superconducting material develop vortex solutions similar to bulk type-II superconductors once the thickness becomes smaller than the penetration depth, as was first observed by Tinkham [30].

3.2. The bulk London Model. Let us now consider the opposite sequence of limits, namely we first let $\Xi \rightarrow 0$ keeping $\lambda$ and $\epsilon$ fixed (i.e. we let $\kappa \rightarrow \infty$ ) and then we let $\epsilon \rightarrow 0$, that is, we are in the parameter regime $\Xi \ll \epsilon \ll 1$.

We have seen that the vortex cores have vanishingly small radius in the limit $\Xi \rightarrow 0$ and (2.25) gives $f=a(\boldsymbol{x})$ except at these isolated vortex lines. Then from $(2.27)$

$$
\frac{\lambda^{2}}{L^{2}} \operatorname{curl}^{2} \boldsymbol{Q}+a \boldsymbol{Q}=\mathbf{0}
$$


or, taking the curl,

$$
\frac{\lambda^{2}}{L^{2}} \operatorname{curl}\left(\frac{1}{a} \operatorname{curl} \boldsymbol{H}\right)+\boldsymbol{H}=\mathbf{0},
$$

away from vortices. By matching a local asymptotic analysis of an individual vortex core with this outer expansion away from vortices, as in Section 3.1 and as detailed in [11], it may be shown that the presence of vortices leads to $\delta$-function singularities on the right-hand side of $(3.54)$, so that

$$
\frac{\lambda^{2}}{L^{2}} \operatorname{curl}\left(\frac{1}{a} \operatorname{curl} \boldsymbol{H}\right)+\boldsymbol{H}=2 \pi \sum_{n} \boldsymbol{\delta}_{\Gamma_{n}},
$$

where

$$
\boldsymbol{\delta}_{\Gamma}(\boldsymbol{x})=\int_{\Gamma} \delta(x-\bar{x}) \delta(y-\bar{y}) \delta(z-\bar{z}) \boldsymbol{d} \overline{\boldsymbol{x}} .
$$

Proceeding to first order in this asymptotic matching and applying a solvability condition gives the law of motion of superconducting vortices as $[11,12]$

$$
\boldsymbol{v}=\frac{|\log \Xi|}{\beta} C \boldsymbol{n}-\frac{|\log \Xi|}{\beta} \nabla \log a+\frac{2}{a \beta} \boldsymbol{J} \wedge \boldsymbol{t},
$$

where $\boldsymbol{J}$ is the background current, $C$ is the curvature of the vortex line, and $\beta$ is given by (3.36) as before.

It has been shown in [10] that (3.57), (3.55) imply that vortices must meet the boundary $\partial \Omega$ normally, since if this is not so an infinite current density is produced (this can also be thought of as an infinite curvature of the vortex and its image, which is clearly incompatible with (3.57)).

Equations (3.55), (3.57) must be coupled with Maxwell's equations (2.6)-(2.10) outside the film, along with continuity conditions on $\boldsymbol{H}$ across $\partial \Omega$.

3.3. Thin film of the London equations. As in Section 2, we assume that the vortex separation is of the same order as the lateral dimension of the film as $\epsilon \rightarrow 0$, that is, the film contains a finite number of vortices in the limit. We write (3.55) as

$$
\begin{aligned}
\boldsymbol{J} & =\epsilon \Lambda \operatorname{curl} \boldsymbol{H}, \\
\operatorname{curl}\left(\frac{\boldsymbol{J}}{a}\right)+\boldsymbol{H} & =2 \pi \sum_{n} \boldsymbol{\delta}_{\Gamma_{n}},
\end{aligned}
$$

where our scaling of $\boldsymbol{J}$, consistent with Section 2, is such that terms in equation (3.59) are balanced. Of course, in the canonical case both equations (3.58) and (3.59) will be balanced, and it is only when we are considering limiting cases that the scaling of $\boldsymbol{J}$ is important. As in Section 2 we will find that the canonical scaling is to have $\Lambda=\lambda^{2} / d L$ of order one. Since the vortices must meet the upper and lower surfaces of the film normally, they must lie in the $z$-direction to leading order. Thus the equations to be satisfied are

$$
\operatorname{curl} \boldsymbol{j}+\boldsymbol{H}=2 \pi \sum_{n} \delta\left(\boldsymbol{x}-\boldsymbol{x}_{n}\right) \boldsymbol{e}_{z}+\cdots \quad \text { in } \Omega,
$$

$$
a \boldsymbol{j}=\Lambda \epsilon \operatorname{curl} \boldsymbol{H} \quad \text { in } \Omega,
$$

$$
\begin{aligned}
\operatorname{div} \boldsymbol{H} & =0 & & \text { in } \Omega, \\
\operatorname{div}(a \boldsymbol{j}) & =0 & & \text { in } \Omega, \\
\operatorname{curl} \boldsymbol{h} & =\boldsymbol{J}_{\text {ext }} & & \text { outside } \Omega, \\
\operatorname{div} \boldsymbol{h} & =0 & & \text { outside } \Omega,
\end{aligned}
$$


with

$$
\begin{array}{rlrl}
\boldsymbol{h} & =\boldsymbol{H} & \text { on } \partial \Omega, \\
\boldsymbol{j} \cdot \boldsymbol{n} & =0 \quad \text { on } \partial \Omega,
\end{array}
$$

and

$$
\boldsymbol{h} \rightarrow \boldsymbol{h}_{\text {ext }} \quad \text { as } \quad|\boldsymbol{r}| \rightarrow \infty
$$

where $\boldsymbol{n}$ is the normal to $\partial \Omega$ and we have written $\boldsymbol{J}=a \boldsymbol{j}$.

As usual, we start by considering the problem inside the film. Rescaling $z=\epsilon Z$ equations (3.60) becomes

$$
\begin{aligned}
-\frac{1}{\epsilon} \frac{\partial j_{2}}{\partial Z}+\frac{\partial j_{3}}{\partial y}+H_{1} & =\cdots, \\
\frac{1}{\epsilon} \frac{\partial j_{1}}{\partial Z}-\frac{\partial j_{3}}{\partial x}+H_{2} & =\cdots, \\
\frac{\partial j_{2}}{\partial x}-\frac{\partial j_{1}}{\partial y}+H_{3} & =2 \pi \sum_{n} \delta\left(\hat{\boldsymbol{x}}-\hat{\boldsymbol{x}}_{n}\right)+\cdots
\end{aligned}
$$

We expand all quantities asymptotically in powers of $\epsilon$ as

$$
\boldsymbol{h} \sim \boldsymbol{h}^{(0)}+\epsilon \boldsymbol{h}^{(1)}+\epsilon^{2} \boldsymbol{h}^{(2)}+\cdots,
$$

etc. Substituting these expansions into (3.69)-(3.71) and equating powers of $\epsilon$ gives, at leading order,

$$
\begin{gathered}
\frac{\partial j_{1}^{(0)}}{\partial Z}=\frac{\partial j_{2}^{(0)}}{\partial Z}=0, \\
\frac{\partial j_{2}^{(0)}}{\partial x}-\frac{\partial j_{1}^{(0)}}{\partial y}+H_{3}^{(0)}=2 \pi \sum_{n} \delta\left(\hat{\boldsymbol{x}}-\hat{\boldsymbol{x}}_{n}\right) .
\end{gathered}
$$

Thus, as before, $j_{1}^{(0)}$ and $j_{2}^{(0)}$ are constant in $Z$. The thin film analysis of (3.61)-(3.68) then proceeds exactly as in Section 2.2 to give [10]

$$
\begin{aligned}
j_{3}^{(0)} & =0, \\
{\left[\boldsymbol{e}_{z} \wedge \boldsymbol{h}^{(0)}\right] } & =\frac{a g}{\Lambda} j^{(0)} \quad \text { for } \quad(x, y) \in D, \\
{\left[\boldsymbol{e}_{z} \cdot \boldsymbol{h}^{(0)}\right] } & =0 \quad \text { for } \quad(x, y) \in D .
\end{aligned}
$$

Similarly the thin film analysis of (3.63) gives

$$
\operatorname{div}\left(a g \boldsymbol{j}^{(0)}\right)=0 .
$$

Finally we need to determine the law of motion for the vortices. An asymptotic analysis in [10] for constant $a$ matching this outer model with the inner model in the vicinity of the vortex cores gives the law of motion

$$
\boldsymbol{v}=\frac{2}{\beta} \boldsymbol{J} \wedge \boldsymbol{e}_{z}-\frac{|\log \Xi|}{\beta} \nabla \log g .
$$

If a variable $a$ is included in this analysis the result is

$$
\boldsymbol{v}=\frac{2}{a \beta} \boldsymbol{J} \wedge \boldsymbol{e}_{z}-\frac{|\log \Xi|}{\beta} \nabla \log (a g) .
$$

It is shown in [10] that the motion due to variations in film thickness is consistent with the motion of the vortex under (3.57) with the curvature it must have if it forms an arc of a circle meeting the upper and lower surfaces of the film normally.

Thus we find that the thin-film version of the London model corresponds exactly to the London limit of the thin-film Ginzburg-Landau model (3.41)-(3.49). 
3.4. Limiting cases. In the limit $\Lambda \rightarrow \infty$ the magnetic field generated by the current in the film gives a negligible contribution to $(3.46)$, and $h_{3}(x, y, 0)$ is simply replaced by $h_{a p p, 3}(x, y, 0)$, the third component of the total applied magnetic field. The problem for the electric current is then decoupled from the problem for the magnetic field, which as usual reduces to the standard problem in electromagnetism of calculating the magnetic field due to a current sheet once the electric current has been found.

For $\Lambda$ small we must rescale the electric current with $\Lambda$. Then the current term in (3.46) is negligible, and this equation reads

$$
h_{3}(x, y, 0)=2 \pi \sum_{n} \delta\left(\boldsymbol{x}-\boldsymbol{x}_{n}\right),
$$

to leading order. Hence the normal component of the magnetic field is zero on $z=0$, except at the positions of the vortices. Near each vortex an inner problem must be solved, in which length is rescaled with $\Lambda$, so that the first term in equation (3.46) is relevant again. Thus, effectively, the relevant horizontal lengthscale is no longer $L$ but the effective penetration depth $\lambda_{\text {eff }}$, and the vortices are completely isolated. This last limit is much more interesting when the vortices are more closely separated, so that the sum of $\delta$-functions can be averaged into a vortex density or vorticity.

4. Vortex-density models. So far we have been considering the limit $\epsilon \rightarrow 0$ with the vortex separation of the same order as $L$, so that there are a finite number of vortices in the film in the limit. In this section we will consider the limit in which $\nu / L \rightarrow 0$ where $\nu$ is the vortex separation, so that the individual vortices are replaced by a vortex density.

As before we now have two limiting processes $(\epsilon \rightarrow 0$ and $\nu / L \rightarrow 0)$, and we can choose to perform them in either order. We will consider first the vortex-density limit of the thin-film London model, that is, we first let $\epsilon \rightarrow 0$ and then let $\nu / L \rightarrow 0$, so that $\epsilon \ll \nu / L \ll 1$. In Section 4.3 we will consider the alternative regime $\nu / L \ll \epsilon \ll 1$.

4.1. Averaging the thin-film London model. We consider the limit in which the vortex separation $\nu / L \rightarrow 0$. In this case it is clear from (3.46) that both $\boldsymbol{h}$ and $\boldsymbol{J}$ need to be rescaled with $L^{2} / \nu^{2}$.

We formally define the vortex density as

$$
\omega(\boldsymbol{x})=\lim _{\eta \rightarrow 0} \frac{2 \pi \nu^{2}}{\eta^{2} L^{2}} \int_{|x|<\eta / 2,|y|<\eta / 2} \sum_{i} \delta\left(\boldsymbol{x}^{\prime}-\boldsymbol{x}_{n}\right) d \boldsymbol{x}^{\prime},
$$

where the integration is over a square of dimension $\eta$, and $\nu / L \ll \eta \ll 1$ as $\nu / L \rightarrow 0$, so that the size of the square is tending to zero, but each square contains many vortices. The prefactor $\nu^{2} / L^{2}$ ensures that the limit is order one. Now locally averaging equations (3.41)-(3.48) by formally integrating over the same square of side $\eta$ we find the electric current inside the superconductor satisfies

$$
\begin{aligned}
\boldsymbol{e}_{z} \cdot \operatorname{curl}\left(\frac{\boldsymbol{J}}{a}\right)+h_{3}(x, y, 0) & =\omega, \\
\operatorname{div}(g \boldsymbol{J}) & =0,
\end{aligned}
$$

with

$$
\boldsymbol{J} \cdot \boldsymbol{\nu}=0 \quad \text { on } \partial D,
$$

while the problem for the magnetic field outside the superconductor,

$$
\begin{aligned}
\operatorname{curl} \boldsymbol{h} & =\boldsymbol{J}_{\text {ext }}, \\
\operatorname{div} \boldsymbol{h} & =0,
\end{aligned}
$$

with

$$
\begin{aligned}
{\left[\boldsymbol{e}_{z} \wedge \boldsymbol{h}\right] } & =\frac{g}{\Lambda} \boldsymbol{J} \quad \text { for } \quad(x, y) \in D, \\
{\left[\boldsymbol{e}_{z} \cdot \boldsymbol{h}\right] } & =0, \\
\boldsymbol{h} & \rightarrow \boldsymbol{h}_{\text {ext }} \quad \text { as } \quad|\boldsymbol{r}| \rightarrow \infty,
\end{aligned}
$$


is unchanged. In the strip geometry, with $a$ and $g$ constant, equation (4.2) was derived by Larkin \& Ovchinnikov [22]. As usual, in this geometry (4.5)-(4.9) can be replaced by (2.124). However, we now have another dependent variable, $\omega$, so we need another equation. In passing from isolated vortices to a vortex density we have lost the property of vortex conservation, which is automatic when we are tracking individual vortices. Thus the equation we need to add is a conservation law for the vortices. This law takes the usual form, namely

$$
\frac{\partial \omega}{\partial t}+\operatorname{div}(\omega \boldsymbol{v})=0
$$

where $\boldsymbol{v}$ is the velocity of the vortices, which is given by (3.49). Remembering that we have rescaled the electric current with $L^{2} / \nu^{2}$ we also now rescale time with $2 L^{2} / \beta \nu^{2}$, to give

$$
\boldsymbol{v}=\frac{1}{a} \boldsymbol{J} \wedge \boldsymbol{e}_{z}-\frac{\nu^{2}|\log \Xi|}{2 L^{2}} \nabla \log (a g)
$$

Now the pinning effect of variations in $a g$ has been weakened due to the increased current density in the film, if the lengthscale for variations in $a g$ is order $L$ then the first term is likely to dominate. In Section 5 we will consider the case when the lengthscale $\varepsilon$ for variations in $a$ is also small, so that pinning becomes important again.

If we perform the same analysis in a region of vortices with negative winding number we find $\omega<0$ and (4.11) becomes

$$
\boldsymbol{v}=-\frac{1}{a} \boldsymbol{J} \wedge \boldsymbol{e}_{z}-\frac{\nu^{2}|\log \Xi|}{2 L^{2}} \nabla \log (a g)
$$

We can combine these two results in the single vortex velocity law

$$
\boldsymbol{v}=\frac{\operatorname{sign}(\omega)}{a} \boldsymbol{J} \wedge \boldsymbol{e}_{z}-\frac{\nu^{2}|\log \Xi|}{2 L^{2}} \nabla \log (a g) .
$$

Equation (4.10) introduces one real characteristic into the model, so that we need to give an extra boundary condition (on $\omega$ ) whenever this characteristic points into the domain, i.e. whenever $\boldsymbol{v} \cdot \boldsymbol{\nu}<0$. This condition will typically relate either the magnitude of the vorticity or the flux of vorticity through the boundary to the local current density. In the two-dimensional case it is found that vortices will not be nucleated at the boundary until the current density reaches a critical value. In the thin-film case the details of vortex nucleation will depend on the local shape of the film near the boundary $\partial D$, and an inner boundary layer problem needs to be solved on a lateral lengthscale of order $d$, the film thickness. However, it is natural to assume still that vortices will not be nulceated until the current density at the boundary reaches a critical value, $J_{\text {nucl }}$ say.

4.2. The bulk vortex-density model. Let us now consider the parameter regime $\nu / L \ll \epsilon \ll 1$, that is, we consider first the limit $\nu / L \rightarrow 0$ to obtain the bulk vortex-density model, and then consider the thin-film limit of it by letting $\epsilon \rightarrow 0$.

In three dimensions a similar formal averaging gives the vortex-density model as [6]

$$
\begin{aligned}
\frac{\lambda^{2}}{L^{2}} \operatorname{curl}\left(\frac{1}{a} \operatorname{curl} \boldsymbol{H}\right)+\boldsymbol{H} & =\boldsymbol{\omega} \quad \text { in } \Omega, \\
\operatorname{div} \boldsymbol{H} & =0,
\end{aligned}
$$

with Maxwells equations (2.6)-(2.10) as usual outside $\Omega$, with continuity of $\boldsymbol{H}$ across $\partial \Omega$ and

$$
\boldsymbol{J} \cdot \boldsymbol{n}=0 \quad \text { on } \partial \Omega,
$$

where $\boldsymbol{\omega}$ is now a vector vortex density or vorticity given by

$$
\boldsymbol{\omega}(\boldsymbol{x})=\lim _{\eta \rightarrow 0} \frac{2 \pi \nu^{2}}{\eta^{2} L^{2}} \int_{|x|<\eta / 2,|y|<\eta / 2} \sum_{i} \boldsymbol{\delta}_{\Gamma_{n}}\left(\boldsymbol{x}^{\prime}\right) d \boldsymbol{x}^{\prime} .
$$


The law of conservation of vortices in three dimensions is $[6,5]$

$$
\frac{\partial \boldsymbol{\omega}}{\partial t}+\operatorname{curl}(\boldsymbol{\omega} \wedge \boldsymbol{v})=0,
$$

where the vortex velocity $\boldsymbol{v}$ is given by

$$
\boldsymbol{v}=\frac{1}{a} \boldsymbol{J} \wedge \hat{\boldsymbol{\omega}}+\frac{\nu^{2}|\log \Xi|}{2 L^{2}}(-\nabla \log a+\operatorname{curl} \hat{\boldsymbol{\omega}} \wedge \hat{\boldsymbol{\omega}}),
$$

and $\hat{\boldsymbol{\omega}}$ is the unit vector in the direction of $\boldsymbol{\omega}$. The term curl $\hat{\boldsymbol{\omega}} \wedge \hat{\boldsymbol{\omega}}$ here is just the curvature term rewritten. If the limits $\Xi \rightarrow 0$ and $\nu / L \rightarrow 0$ are such that $\nu^{2}|\log \Xi| / L^{2} \rightarrow 0$ then the last term is of lower order and can be neglected. In the general three-dimensional situation neglecting the self-induced curvature term leads to the possibility of a short-wavelength large-growth-rate instability on vortex lines if $\boldsymbol{J} \cdot \boldsymbol{\omega} \neq 0$, as shown in [26] and discussed in [6]. However, in the thin-film limit it is safe to neglect the self-induced term since the current will lie in the $(x, y)$-plane while the vorticity will be normal to it.

As in the thin-film vortex-density model, equation (4.17) has introduced one real characteristic, and we need to give a boundary condition on $\boldsymbol{\omega}$ whenever $\boldsymbol{v} \cdot \boldsymbol{n}<0$. Now, an analysis in [15] indicates that on such an inflow boundary either $\boldsymbol{\omega} \cdot \boldsymbol{n}=0$ (which is the case in the thin-film model and also in the two-dimensional model with axial symmetry) or $\boldsymbol{\omega} \wedge \boldsymbol{n}=\mathbf{0}$. In the first case the flux of vorticity through the boundary will be related to the local current density, while in the second no extra condition needs to be given. In a general three-dimensional situation the position of the switch between these two types of behaviour is unknown, and a boundary layer calculation may be necessary in its vicinity. However, in our thin film scenario we are fortunate that the top and bottom of the film will have vortices passing through them and be such that $\boldsymbol{\omega} \wedge \boldsymbol{n}=\mathbf{0}$ if they are inflow, while we will see that the sides of the film will have $\boldsymbol{\omega} \cdot \boldsymbol{n}=0$ to leading order, and therefore these are the boundaries through which new vortices will pass.

4.3. Thin film of the vortex-density model. Let us now consider the thin-film limit $\epsilon \rightarrow 0$ of the bulk vortex density model (4.14)-(4.18), corresponding to the parameter regime $\nu / L \ll \epsilon \ll 1$. We begin as usual by writing (4.14) as

$$
\begin{aligned}
\operatorname{curl}\left(\frac{\boldsymbol{J}}{a}\right)+\boldsymbol{H} & =\boldsymbol{\omega} \quad \text { in } \Omega, \\
\boldsymbol{J} & =\Lambda \epsilon \operatorname{curl} \boldsymbol{H} \quad \text { in } \Omega,
\end{aligned}
$$

Expanding all quantities in powers of $\epsilon$ the analysis proceeds exactly as in Section (3.3) giving

$$
\begin{aligned}
\operatorname{curl} \boldsymbol{h} & =\boldsymbol{J}_{\text {ext }}, \\
\operatorname{div} \boldsymbol{h} & =0,
\end{aligned}
$$

with

$$
\begin{aligned}
{\left[\boldsymbol{e}_{z} \wedge \boldsymbol{h}\right] } & =\frac{g}{\Lambda} \boldsymbol{J} \quad \text { for } \quad(x, y) \in D, \\
{\left[\boldsymbol{e}_{z} \cdot \boldsymbol{h}\right] } & =0, \\
\boldsymbol{h} & \rightarrow \boldsymbol{h}_{\text {ext }} \quad \text { as } \quad|\boldsymbol{r}| \rightarrow \infty,
\end{aligned}
$$

for the magnetic field outside the superconductor, and

$$
\begin{aligned}
\boldsymbol{e}_{z} \cdot \operatorname{curl}\left(\frac{\boldsymbol{J}}{a}\right)+h_{3}(x, y, 0) & =\omega_{3}, \\
\operatorname{div}(g \boldsymbol{J}) & =0,
\end{aligned}
$$

with

$$
\boldsymbol{J} \cdot \boldsymbol{\nu}={ }_{21} \text { on } \partial D,
$$


for the electric current inside the superconductor. The only additional work is to check that the threedimensional vector conservation law (4.17) reduces to the two-dimensional scalar conservation law (4.10) in the thin-film limit. To do that we need to show that $\boldsymbol{\omega}$ lies in the $z$-direction to leading order. Since one of the top or bottom sides of the film is an inflow boundary, and on that inflow boundary $\boldsymbol{\omega} \wedge \boldsymbol{n}=\mathbf{0}$, to leading order we must have $\boldsymbol{\omega} \wedge \boldsymbol{e}_{z}=0$ there. To be sure that $\omega_{1}=\omega_{2}=0$ everywhere we must check that they cannot vary rapidly in $z$, i.e. they are independent of $Z$ to leading order. Writing (4.17) in component form in the film gives

$$
\begin{gathered}
\frac{\partial \omega_{1}}{\partial t}+\frac{\partial}{\partial y}\left(\omega_{1} v_{2}-\omega_{2} v_{1}\right)-\frac{1}{\epsilon} \frac{\partial}{\partial Z}\left(\omega_{3} v_{1}-\omega_{1} v_{3}\right)=0 \\
\frac{\partial \omega_{2}}{\partial t}-\frac{\partial}{\partial x}\left(\omega_{1} v_{2}-\omega_{2} v_{1}\right)+\frac{1}{\epsilon} \frac{\partial}{\partial Z}\left(\omega_{2} v_{3}-\omega_{3} v_{2}\right)=0 \\
\frac{\partial \omega_{3}}{\partial t}+\frac{\partial}{\partial x}\left(\omega_{3} v_{1}-\omega_{1} v_{3}\right)-\frac{\partial}{\partial y}\left(\omega_{2} v_{3}-\omega_{3} v_{2}\right)=0
\end{gathered}
$$

where

$$
\begin{aligned}
& a v_{1}=J_{2} \hat{\omega}_{3}, \\
& a v_{2}=-J_{1} \hat{\omega}_{3}, \\
& a v_{3}=J_{1} \hat{\omega}_{2}-J_{2} \hat{\omega}_{1} .
\end{aligned}
$$

Thus at leading order in $\epsilon$

$$
\begin{aligned}
& \omega_{3} v_{1}-\omega_{1} v_{3}=\text { independent of } Z, \\
& \omega_{2} v_{3}-\omega_{3} v_{2}=\text { independent of } Z .
\end{aligned}
$$

Now (4.19) implies div $\boldsymbol{\omega}=0$, which in the film gives $\omega_{3}$ independent of $Z$ to leading order. Then eliminating $\boldsymbol{v}$ from (4.35)-(4.36) gives two equations for $\omega_{1}$ and $\omega_{2}$ in terms of $\omega_{3}$, so that $\omega_{1}$ and $\omega_{2}$, and therefore $\boldsymbol{v}$, are also independent of $Z$ to leading order.

Finally (4.31) then gives

$$
\frac{\partial \omega_{3}}{\partial t}+\frac{\partial}{\partial x}\left(\omega_{3} v_{1}\right)+\frac{\partial}{\partial y}\left(\omega_{3} v_{2}\right)=0
$$

as required.

4.4. Limiting cases. In the limit in which $\Lambda \rightarrow \infty$, the problem for the electric current becomes

$$
\begin{aligned}
\boldsymbol{e}_{z} \cdot \operatorname{curl}\left(\frac{\boldsymbol{J}}{a}\right)+h_{a p p, 3}(x, y, 0) & =\omega, \\
\frac{\partial \omega}{\partial t}+\operatorname{div}(\omega \boldsymbol{v}) & =0, \\
\boldsymbol{v} & =\frac{\operatorname{sign}(\omega)}{a} \boldsymbol{J} \wedge \boldsymbol{e}_{z}, \\
\operatorname{div}(g \boldsymbol{J}) & =0,
\end{aligned}
$$

with

$$
\boldsymbol{J} \cdot \boldsymbol{\nu}=0 \quad \text { on } \quad \partial \Omega .
$$

Once the current has been found, the magnetic field may be found from (4.5)-(4.9) in the usual way.

In the limit in which $\Lambda \rightarrow 0$ we must scale the electric current with $\Lambda$ to give

$$
\begin{aligned}
\operatorname{curl} \boldsymbol{h} & =\boldsymbol{J}_{\text {ext }}, \\
\operatorname{div} \boldsymbol{h} & =0,
\end{aligned}
$$


with

$$
\begin{gathered}
{\left[\boldsymbol{e}_{z} \wedge \boldsymbol{h}\right]=g \boldsymbol{J} \quad \text { for } \quad(x, y) \in \Omega,} \\
{\left[\boldsymbol{e}_{z} \cdot \boldsymbol{h}\right]=0,} \\
\boldsymbol{h}^{(0)} \rightarrow \boldsymbol{h}_{\text {ext }} \quad \text { as } \quad|\boldsymbol{r}| \rightarrow \infty, \\
h_{3}(x, y, 0)=\omega .
\end{gathered}
$$

The problems for the magnetic field and electric current again simplify, but in this case equations (4.43)(4.44), (4.46)-(4.48) are first solved for the magnetic field, and then equation (4.45) is used to determine the current in the superconductor. Having found the current equations (4.10) and (4.13) are then solved for the vortex density, which in turn feeds into (4.48). Thus the problems remain coupled in this limit.

However, in taking the limit $\nu / L \rightarrow 0$ and $\Lambda \rightarrow 0$ there is a constraint on relative magnitudes of these two parameters for the model (4.10) (4.13), (4.43)-(4.48) to be valid. As we let $\Lambda \rightarrow 0$ we are weakening the size of the mean-field current over the local perturbation to this current due to neighbouring individual vortices. We have seen that the mean-field current is $O\left(\Lambda L^{2} / \nu^{2}\right)$, while influence of the current due to a single vortex on its neighbours is $O(L / \nu)$. Thus for the dynamics to de dominated by the mean field as in (4.13) we need $\nu / L \ll \Lambda$, i.e. $\nu \ll \lambda_{\text {eff }}$. If this is not the case then the vortex motion will be dominated by local forces and they will form a strong lattice, rather than the vortex liquid we are supposing. Note that for bulk superconductors the equivalent condition is $\nu<\lambda^{2} / L$; the thin film condition is much less stringent since the right-hand side is increased by the aspect ratio $L / d$.

In the strip geometry (4.10) (4.13), (4.43)-(4.48) reduce to an interesting singular integral equation. Suppose that $D=(x, y):-l<x<l$ and that $h_{a p p, 2}=0$ as usual. In the strip geometry vortex conservation (4.39) gives

$$
\frac{\partial \omega}{\partial t}+\frac{\partial}{\partial x}\left(\frac{J_{2}|\omega|}{a}\right)=0,
$$

while (2.124) and (4.48) give

$$
\omega-h_{a p p, 3}=\frac{1}{\pi} \int_{-l}^{l} \frac{g J_{2}(\bar{x})}{2(\bar{x}-x)} d \bar{x}, \quad-l<x<l .
$$

Hence

$$
\frac{\partial}{\partial t}\left(h_{a p p, 3}+\frac{1}{\pi} \int_{-l}^{l} \frac{g J_{2}(\bar{x})}{2(\bar{x}-x)} d \bar{x}\right)+\frac{\partial}{\partial x}\left(\frac{J_{2}}{a}\left|h_{a p p, 3}+\frac{1}{\pi} \int_{-l}^{l} \frac{g J_{2}(\bar{x})}{2(\bar{x}-x)} d \bar{x}\right|\right)=0, \quad-l<x<l .
$$

If $l=\infty$ we may invert (4.50) to give

$$
\frac{g J_{2}}{2}=\frac{1}{\pi} \int_{-\infty}^{\infty} \frac{\omega(\bar{x})-h_{a p p, 3}}{x-\bar{x}} d \bar{x} .
$$

and hence

$$
\frac{\partial \omega}{\partial t}+\frac{\partial}{\partial x}\left(\frac{2|\omega|}{a g}\left[\frac{1}{\pi} \int_{-\infty}^{\infty} \frac{\omega(\bar{x})-h_{a p p, 3}}{x-\bar{x}} d \bar{x}\right]\right)=0 .
$$

This inversion is not so easy if $l$ is finite since $J_{2}$ depends on $h_{3}$ outside the domain $D$, which cannot be simply written in terms of $\omega$.

5. Critical-state models. So far we have been considering the limit in which the separation of vortices $\nu / L \rightarrow 0$ but in which the lengthscale for variations in $a$, namely $\varepsilon$, remains fixed. Let us now consider the limit in which $\varepsilon / L \rightarrow 0$ also, so that the pinning potential is also homogenised. Such a limit will lead to critical state models, in which vortices do not move until the forcing current exceeds a critical value. 

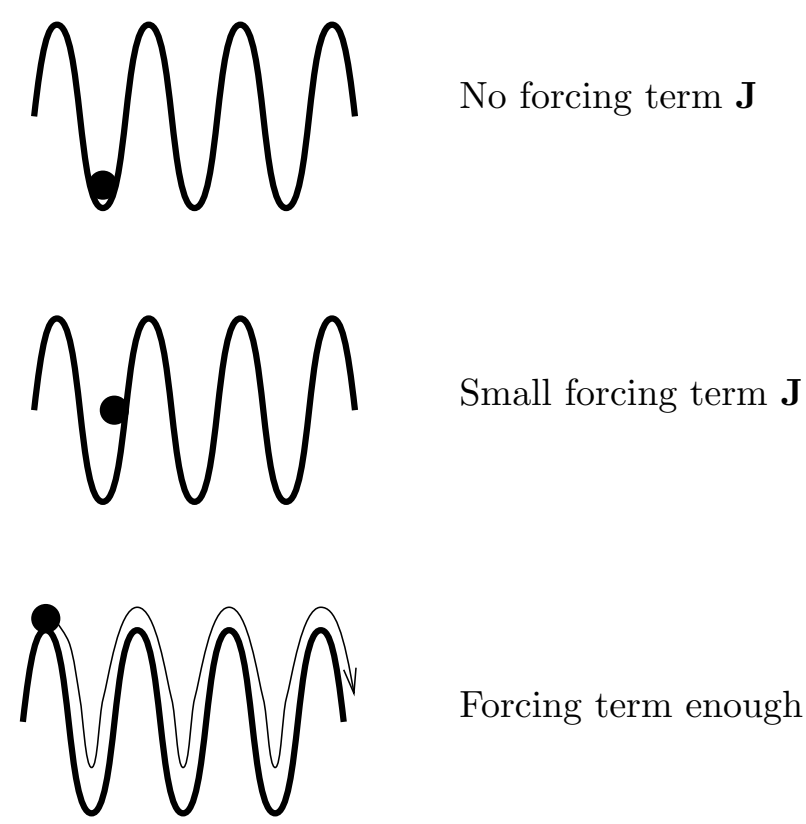

Forcing term enough to depin

FIG. 5.1. Pinning of a single vortex by a rapidly varying pinning potential.

5.1. Critical-state limit of the vortex-density thin-film model. Suppose we now allow $a$ in (4.2)(4.11) vary rapidly. If we look locally near a single vortex which is in the deepest local well and consider the effect of applying a current $\boldsymbol{J}$ to it we see that if the current is not sufficient to cause the vortex to leave its local well, then it will move up the side of the well until the attraction from the potential balances the applied current (see Figure 5.1). In this case the vortex will have moved a distance of order $\varepsilon$. Now, if we increase the current $\boldsymbol{J}$ then at some point it will be sufficient to cause the vortex to leave its local well and it will jump into the next well. However, since the vortex was in the deepest local well the current will also be sufficient to cause the vortex to leave the neighbouring well. It will continue in this way until it has moved an order-one distance, until either the local well depth has increased, or until the local current density has decreased sufficiently to catch the vortex. Hence, in the limit that $\varepsilon / L \rightarrow 0$, we arrive at a stick-slip mobility law: if the local current density is less that a critical value, $J_{c}$ say (which may be a function of position), then the vortex does not move, while if the local current density is greater than than $J_{c}$ then the vortex will move, but at a reduced speed due to moving through the potential wells. If the distribution of well depth is nonuniform, so that there are a range of depths locally, then it is possible to see how the critical current in this stick-slip model may depend on $\omega$. If we add more and more vortices locally we are having to make use of shallower and shallower wells, thus reducing the critical current required to start some of the vortices moving.

The critical current density in these scalings will be of order $\left(\nu^{2} / L \varepsilon\right)|\log \Xi|$.

We have described the interaction of a single vortex with a rapidly varying potential, which is the case $\varepsilon \ll \nu$. A similar scenario exists in the complementary situation $\nu \ll \varepsilon$ with the single vortex replaced by a "pool" of vorticity, as shown in Figure 5.2. Applying a forcing term now corresponds to "tilting" the potential, and again there will be a critical current at which the well can no longer hold the pool. In this case it is clear that the critical current will depend on the vortex density.

Thus, after homogenising the pinning potential the law of motion (4.13) is modified to be

$$
\boldsymbol{v}=\operatorname{sign}(\omega) F(|\boldsymbol{J}| ; \boldsymbol{x}, \omega) \hat{\boldsymbol{J}} \wedge \boldsymbol{e}_{z}
$$

where $\hat{\boldsymbol{J}}$ is the unit vector in the direction of $\boldsymbol{J}$, and $F(J)=0$, for $J<J_{c}(\boldsymbol{x}, \omega)$ (see Figure 5.3). The value of $J_{c}$ and the exact form of the velocity law for $J$ above $J_{c}$ depend on the nature of the pinning potential. We do not devle into the details of this correspondence here, but note that for a single vortex moving in a simple sinosoidal potential with uniform well depth $F$ approaches zero as $\left(J-J_{c}\right)^{1 / 2}$. Note also that (4.2) 

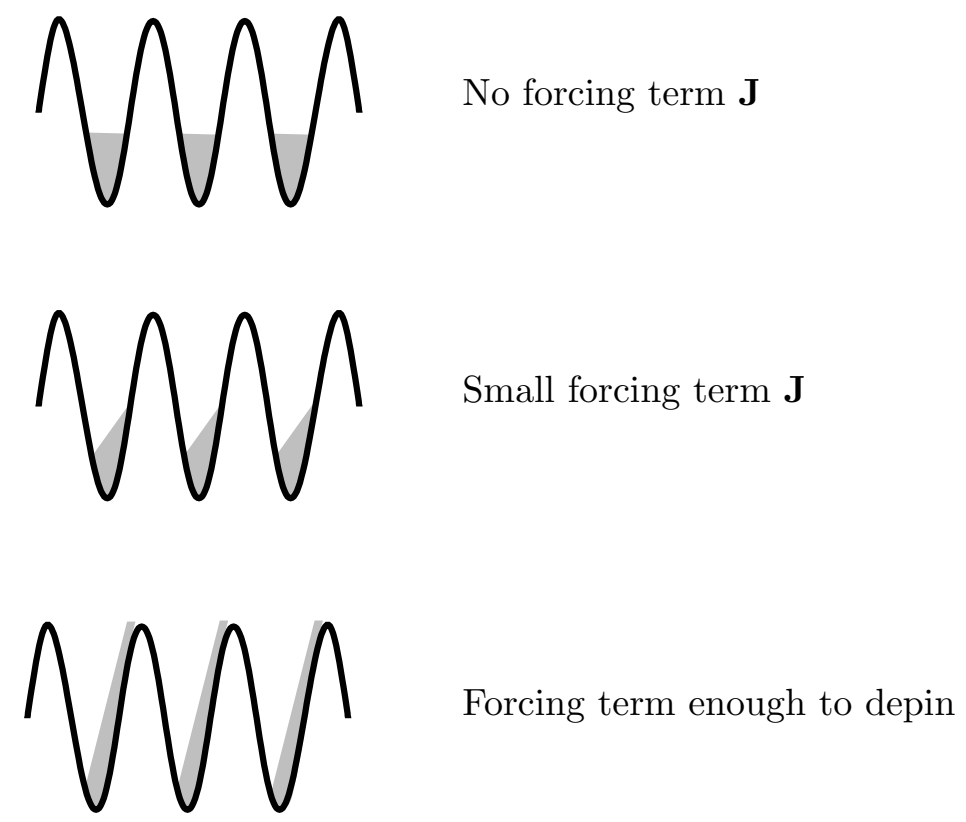

FIG. 5.2. Pinning of a vortex pool by a rapidly varying pinning potential.

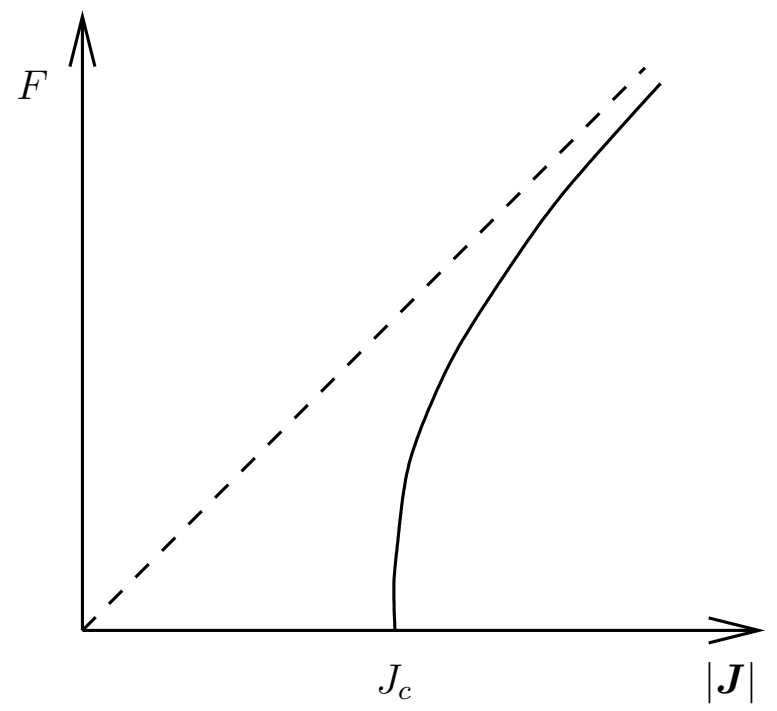

FIG. 5.3. Velocity law with stick-slip pinning.

becomes

$$
\boldsymbol{e}_{z} \cdot \operatorname{curl}\left(\frac{1}{\hat{a}(\boldsymbol{x})} \boldsymbol{J}\right)+h_{3}(x, y, 0)=\omega,
$$

where $\hat{a}$ is the effective equilibrium density of superconducting electrons, which is not simply the local average of $a$, but must be determined through a multiple scales analysis.

Since the critical state corresponds to simply changing the law of motion, the thin-film limit of the bulk 
critical-state model leads to the same set of equations.

We note that it is quite common in the literature to use a vortex velocity law in which the velocity depends on the electric current via a power law, either with or without a critical current. In [9] a law of this type is used to derive the current-voltage characteristics of thin strips from the underlying vortex velocity law. Even more common (especially when $\Lambda=0$ ) is to model the superconductor as a nonlinear conductor by assuming a nonlinear (typically power-law) relationship between the electric field

$$
\boldsymbol{E}=\Lambda \frac{\partial \boldsymbol{J}}{\partial t}+\omega \boldsymbol{e}_{z} \wedge \boldsymbol{v}=\Lambda \frac{\partial \boldsymbol{J}}{\partial t}+|\omega| F(|\boldsymbol{J}| ; \boldsymbol{x}, \omega) \hat{\boldsymbol{J}}
$$

and the electric current. When $\Lambda=0$ this corresponds to choosing $F(|\boldsymbol{J}| ; \boldsymbol{x}, \omega) \propto|\boldsymbol{J}|^{n} /|\omega|$. Typically the power chosen is large, $n=9$ [28] or even $n=19$ [29]. When $n$ is large these power laws approximate to a stick-slip law of the form (5.1), but with an infinite vortex mobility once the critical current is exceeded (so that the graph in Figure 5.3 is vertical). Such an infinite mobility also approximates an arbitrary stick slip law (5.1) when the applied magnetic field is slowly varying.

When the mobility of unpinned vortices is infinite the velocity $\boldsymbol{v}$ lies in the direction of $\operatorname{sign}(\omega) \boldsymbol{J} \wedge \boldsymbol{e}_{z}$, but its magnitude is determined from the constraint that $\left|\boldsymbol{J}^{(0)}\right| \leq J_{c}$, so that

$$
\begin{gathered}
\boldsymbol{v}=\operatorname{sign}(\omega) m \boldsymbol{J} \wedge \boldsymbol{e}_{z}, \\
|\boldsymbol{J}| \leq J_{c}, \text { if } \omega \neq 0, \\
m \geq 0, \\
m\left(|\boldsymbol{J}|-J_{c}\right)=0 .
\end{gathered}
$$

These equations are coupled with the law of vortex conservation

$$
\frac{\partial \omega}{\partial t}+\operatorname{div}(\omega \boldsymbol{v})=0,
$$

and the usual equations for the magnetic field and electric current, namely

$$
\begin{aligned}
\operatorname{curl} \boldsymbol{h} & =\boldsymbol{J}_{e x t}, \\
\operatorname{div} \boldsymbol{h} & =0,
\end{aligned}
$$

with

$$
\begin{aligned}
{\left[\boldsymbol{e}_{z} \wedge \boldsymbol{h}\right] } & =\frac{g}{\Lambda} \boldsymbol{J} \quad \text { for } \quad(x, y) \in D, \\
{\left[\boldsymbol{e}_{z} \cdot \boldsymbol{h}\right] } & =0, \\
\boldsymbol{h} & \rightarrow \boldsymbol{h}_{\text {ext }} \quad \text { as } \quad|\boldsymbol{r}| \rightarrow \infty
\end{aligned}
$$

and

$$
\begin{aligned}
\boldsymbol{e}_{z} \cdot \operatorname{curl}\left(\frac{\boldsymbol{J}}{\hat{a}}\right)+h_{3}(x, y, 0) & =\omega, \\
\operatorname{div}(g \boldsymbol{J}) & =0,
\end{aligned}
$$

with

$$
\boldsymbol{J} \cdot \boldsymbol{\nu}=0 \quad \text { on } \partial D
$$

However, so far $\omega=0$ is a solution for all applied magnetic fields. What we are missing is the condition of nucleation of vortices at the boundary $\partial D$. Vortices will be nucleated once the current reaches the nucleation value $J_{\text {nucl }}$, but if $J_{\text {nucl }}<J_{c}$ they will not move until the current reaches this higher depinning value. Thus in addition to (5.4) we must also impose

$$
|\boldsymbol{J}| \leq J_{c} \quad \text { on } \partial D .
$$


Now in three dimensions in regions where $\omega=0$, taking the curl of (4.19) shows that $|\boldsymbol{J}|$ takes its maximum value on the boundary. If this is true for the thin film model (5.13) also then we can replace (5.3)-(5.6) with

$$
\begin{gathered}
\omega \boldsymbol{v}=m \boldsymbol{J} \wedge \boldsymbol{e}_{z}, \\
|\boldsymbol{J}| \leq J_{c}, \quad m \geq 0, \quad m\left(|\boldsymbol{J}|-J_{c}\right)=0 .
\end{gathered}
$$

Streamfunction formulation. Using (5.14) we may introduce a streamfunction $\psi$ such that

$$
\boldsymbol{J}=\frac{1}{g}\left(\psi_{y},-\psi_{x}, 0\right) .
$$

Then $\boldsymbol{v}$ is in the direction of $-\nabla \psi$, so we may set

$$
\omega \boldsymbol{v}=-m \nabla \psi,
$$

giving

$$
\begin{aligned}
\frac{\partial \omega}{\partial t} & =\operatorname{div}(m \nabla \psi), \\
-\nabla \cdot\left(\frac{1}{\hat{a} g} \nabla \psi\right)+h_{3}(x, y, 0) & =\omega,
\end{aligned}
$$

with the constraints

$$
|\nabla \psi| \leq g J_{c}, \quad m \geq 0, \quad m\left(|\nabla \psi|-g J_{c}\right)=0 .
$$

These equations are coupled to (5.8)-(5.12) for $\Lambda$ order one.

In a virgin sample in an increasing magnetic field there will be a region in the interior, $D_{1}$ say, in which vortices have not yet reached, and a region around the boundary, $D_{2}=D \backslash D_{1}$ say, in which the current is equal to the critical value.

In a strip $-l<x<l$, with $\hat{a}=1$ and $J_{c}$ constant, and with $D_{1}$ given by $-s<x<s$, this gives

$$
\begin{aligned}
\frac{\partial J_{2}}{\partial x}+h_{3}(x, 0) & =0 \quad \text { for }|x|<s, \\
J_{2} & =J_{c} \quad \text { for }-l<x<-s, \\
J_{2} & =-J_{c} \quad \text { for } s<x<l,
\end{aligned}
$$

with $J_{2}$ continuous at $x= \pm s$, and

$$
\begin{aligned}
& \omega=0 \quad \text { for }|x|<s, \\
& \omega=h_{3}(x, 0) \quad \text { for } s<|x|<l,
\end{aligned}
$$

where

$$
h_{3}(x, 0)-h_{a p p, 3}(x, 0)=\frac{1}{\pi} \int_{-l}^{l} \frac{g J_{2}(\bar{x})}{2 \Lambda(\bar{x}-x)} d \bar{x} .
$$

5.2. Limiting Cases. As $\Lambda \rightarrow \infty$ the problems for the current decouples from the problem for the magnetic field, since, as usual, $h_{3}(x, y, 0)$ in $(5.22)$ is simply replaced by $h_{a p p, 3}(x, y, 0)$. Then we have

$$
\begin{aligned}
\frac{\partial \omega}{\partial t} & =\operatorname{div}(m \nabla \psi), \\
-\nabla \cdot\left(\frac{1}{\hat{a} g} \nabla \psi\right)+h_{a p p, 3} & =\omega,
\end{aligned}
$$




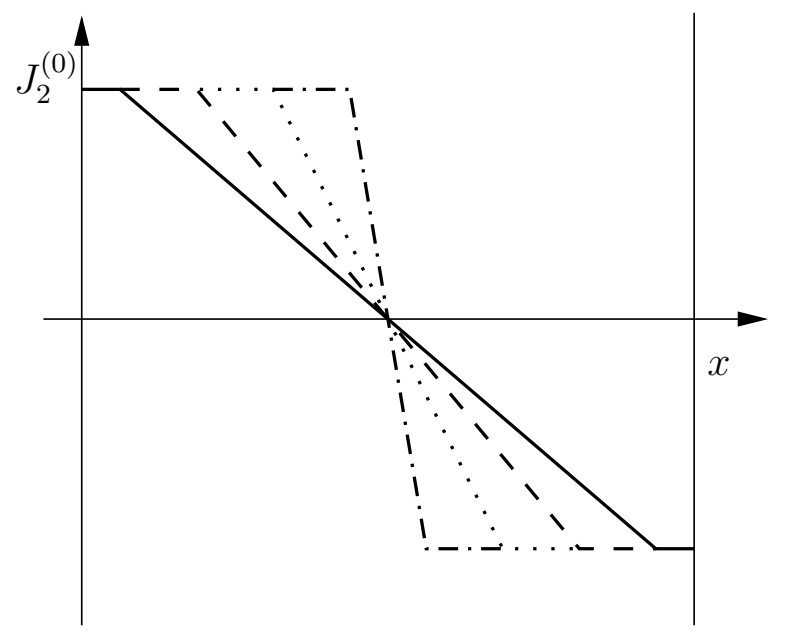

(a)

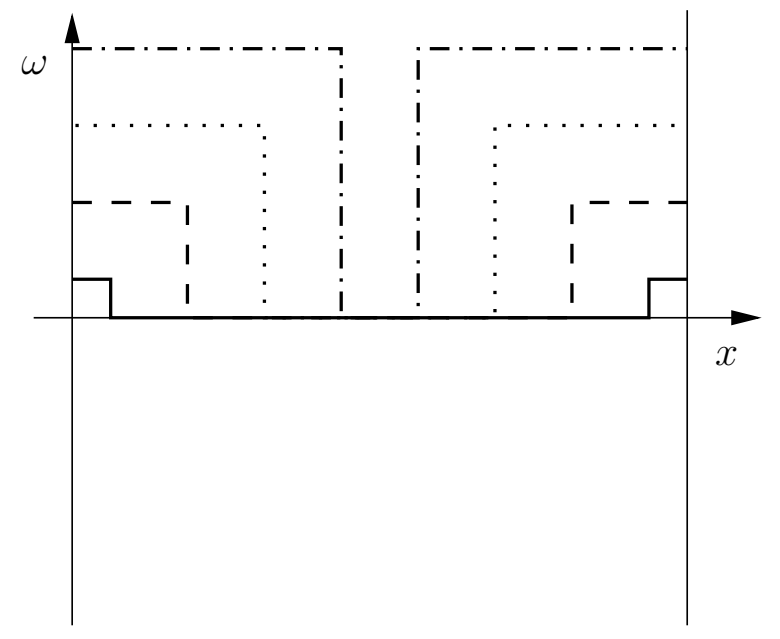

(b)

FIG. 5.4. Solutions of the thin-film critical-state model in a thin strip in the limit $\Lambda \rightarrow \infty$.

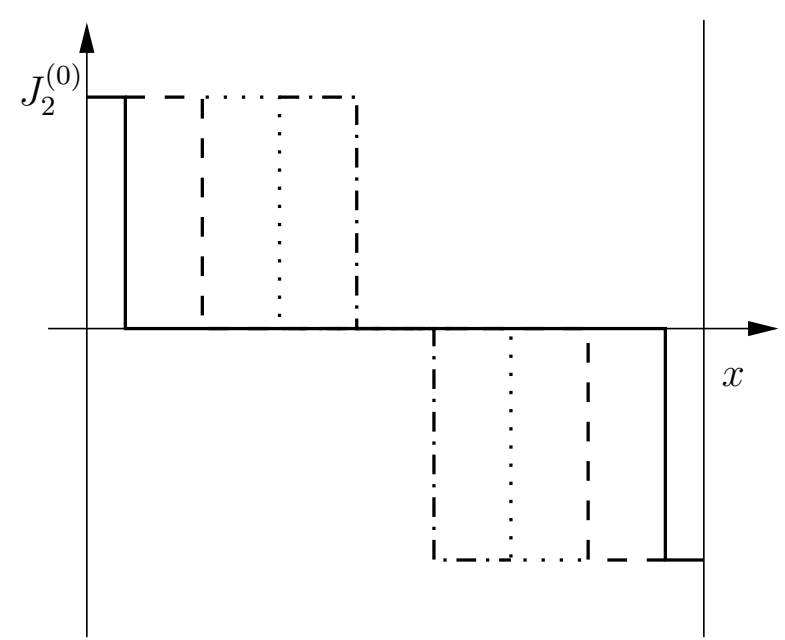

(a)

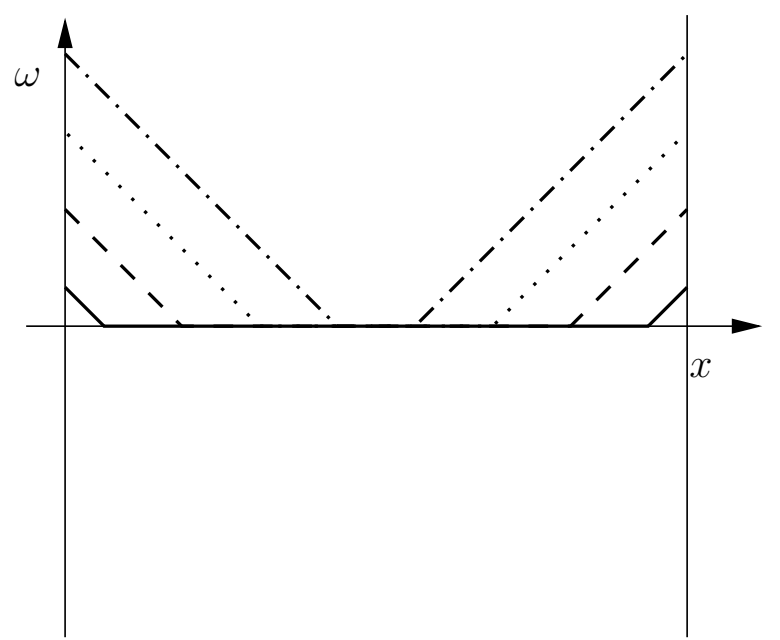

(b)

FIG. 5.5. Solutions of the two-dimensional critical-state model in a slab.

with the constraints

$$
|\nabla \psi| \leq g J_{c}, \quad m \geq 0, \quad m\left(|\nabla \psi|-g J_{c}\right)=0
$$

In this limit with $g=1$ the free-boundary problem (5.24)-(5.26) gives

$$
\begin{aligned}
J_{2} & =J_{c} \quad \text { for }-L<x<-s, \\
J_{2} & =-h_{a p p, 3} x \quad \text { for }-s<x<s, \\
J_{2} & =-J_{c} \quad \text { for } s<x<L, \\
s & =\frac{J_{c}}{h_{a p p, 3}},
\end{aligned}
$$


with

$$
\begin{aligned}
& \omega=0 \quad \text { for }|x|<s, \\
& \omega=h_{\text {app }, 3}(x, y, 0) \quad \text { for } s<|x|<l .
\end{aligned}
$$

These solutions are plotted in Figure 5.4 for an increasing applied magnetic field $h_{a p p, 3}$.

For comparison, the corresponding solutions for a two-dimensional critical-state model in a slab are

$$
\begin{aligned}
J_{2} & =J_{c} \quad \text { for }-L<x<-s, \\
J_{2} & =0 \quad \text { for }-s<x<s, \\
J_{2} & =-J_{c} \quad \text { for } s<x<L, \\
s & =l-\frac{h_{a p p, 3}}{J_{c}}
\end{aligned}
$$

with

$$
\begin{aligned}
& \omega=0 \quad \text { for }|x|<s, \\
& \omega=J_{c}(x-s) \quad \text { for } s<x<l, \\
& \omega=-J_{c}(x+s) \text { for }-l<x<-s,
\end{aligned}
$$

which are shown in Figure 5.5.

In the second limit, $\Lambda \rightarrow 0$ we must again scale $\boldsymbol{J}$ with $\Lambda$ to give

$$
\begin{aligned}
\operatorname{curl} \boldsymbol{h} & =\boldsymbol{J}_{\text {ext }}, \\
\operatorname{div} \boldsymbol{h} & =0,
\end{aligned}
$$

with

$$
\begin{aligned}
& {\left[\boldsymbol{e}_{z} \wedge \boldsymbol{h}\right]=}\left(\psi_{y},-\psi_{x}, 0\right) \quad \text { for } \quad(x, y) \in D, \\
& {\left[\boldsymbol{e}_{z} \cdot \boldsymbol{h}\right]=0, } \\
& \boldsymbol{h} \rightarrow \boldsymbol{h}_{\text {ext }} \quad \text { as } \quad|\boldsymbol{r}| \rightarrow \infty, \\
& \\
& h_{3}(x, y, 0)=\omega . \\
& \frac{\partial \omega}{\partial t}=\operatorname{div}(m \nabla \psi),
\end{aligned}
$$

with the constraints

$$
|\nabla \psi| \leq g J_{c}, \quad m \geq 0, \quad m\left(|\nabla \psi|-g J_{c}\right)=0 .
$$

In this case with $g=1$ the free-boundary problem (5.24)-(5.26) becomes

$$
\begin{aligned}
h_{3}(x, 0) & =h_{a p p, 3}+\frac{1}{\pi} \int_{-l}^{l} \frac{J_{2}(\bar{x})}{2(\bar{x}-x)} d \bar{x}=0 \quad \text { for }|x|<s, \\
J_{2} & =J_{c} \quad \text { for }-l<x<-s, \\
J_{2} & =-J_{c} \quad \text { for } s<x<l .
\end{aligned}
$$

This problem has been considered previously in $[4,10,29,31]$. Solutions are plotted in Figure 5.6. Note that the magnetic field tends to infinity as $x \rightarrow \pm l$; there is an inner region of width $\Lambda$ in which the problems for $\boldsymbol{J}$ and $\boldsymbol{h}$ are coupled again, and which regularises the magnetic field.

In both [4] and [31] field and current profiles are calculated for superconducting strips in an applied field, with an applied current, and with both an applied field and an applied transport current. In [29] the 


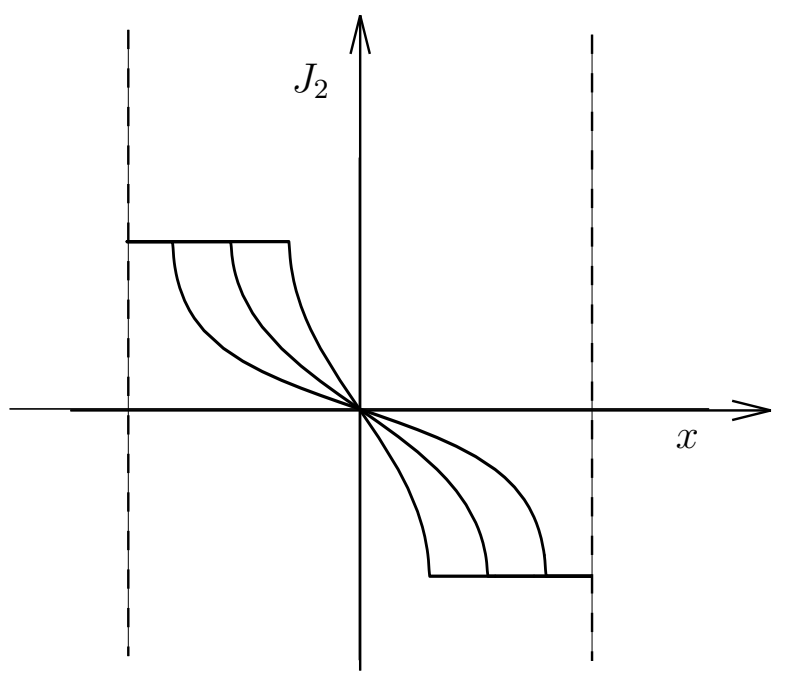

(a)

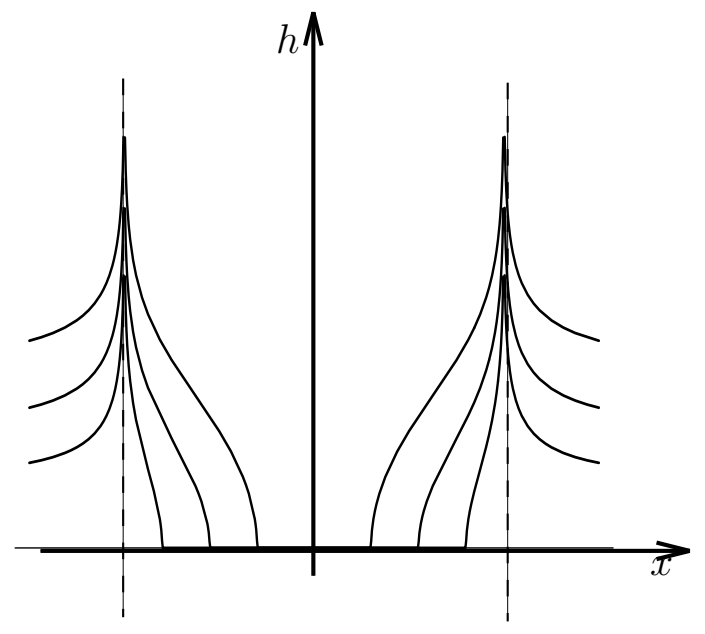

(b)

FIG. 5.6. Solutions of the thin-film critical-state model in a thin strip in the limit $\Lambda \rightarrow 0$.

theoretical profiles are compared with experimentally measured profiles of the magnetic field distribution in a superconducting slab in an applied magnetic field, and the agreement is shown to be good. Schuster et al. also consider experimentally the effects of inhomogeneous pinning. If the critical current is lower in the centre of a sample than it is at the outside, there can be a sudden jump in the free boundary as the applied magnetic field reaches a critical value. This effect is also examined in [20], where its implications for the flux flow regime under an applied current are considered.

In [29] the profiles of magnetic field distribution on a square film in an applied field are measured experimentally, and in [28] the profiles on a film in the shape of a cross are measured experimentally and calculated theoretically, using a power-law dependence of $\boldsymbol{E}$ on $\boldsymbol{J}$. In [20] field and current distributions for general two-dimensional films are calculated numerically in both the critical state and flux flow regimes (using general vortex velocity laws as well as general nonlinear Ohm's laws), both with an applied magnetic field and a transport current. This work also includes the heating effect of the electric field coupled with an equation for the evolution of temperature, with temperature dependent critical fields and vortex mobilities, but a discussion of this extension is beyond the scope of the present paper.

The solutions for a cross in an increasing applied magnetic field are reproduced in figure 5.8. The field is ramped up from zero to 1.5 over 4 time units and then held fixed until the sample reaches steady state, as illustrated in figure 5.7. Figure 5.8 shows the current, vorticity and electric field at times $t=1,2,3$ and 4. Figure 5.9 shows the current and vorticity in the steady state which is reached at about $t=8$ (the electric field is zero in steady state). In figures $5.8(\mathrm{a}, \mathrm{d}, \mathrm{g}, \mathrm{j})$ and $5.9(\mathrm{a})$ the shading shows the magnitude of the current density and the lines show the direction of current flow. In the other figures the lines are contour lines of constant $\omega$ or $|\boldsymbol{E}|$.

The vortices enter first at the reentrant corners of the cross, and this is where the electric field is largest. In the thermal problem the heating at these points may significantly affect the solution.

6. Conclusion. We have developed a hierarchy of models of superconducting thin films, allowing for a spatially varying film thickness and a spatially varying equilibrium density of superconducting electrons.

We began with the Ginzburg-Landau model, where we found that the canonical scaling was to let $\epsilon=d / L \rightarrow 0$ with $\Xi=\xi / L$ and $\Lambda=\lambda_{\text {eff }} / L=\lambda^{2} / d L$ fixed, where $\lambda_{\text {eff }}$ is the effective screening length. This corresponds to letting the Ginzburg-Landau parameter $\kappa=\lambda / \xi$ tend to zero like $\epsilon^{1 / 2}$. The London limit of this model, in which vortices appear as $\delta$-function singularities, corresponds to the limit in which 


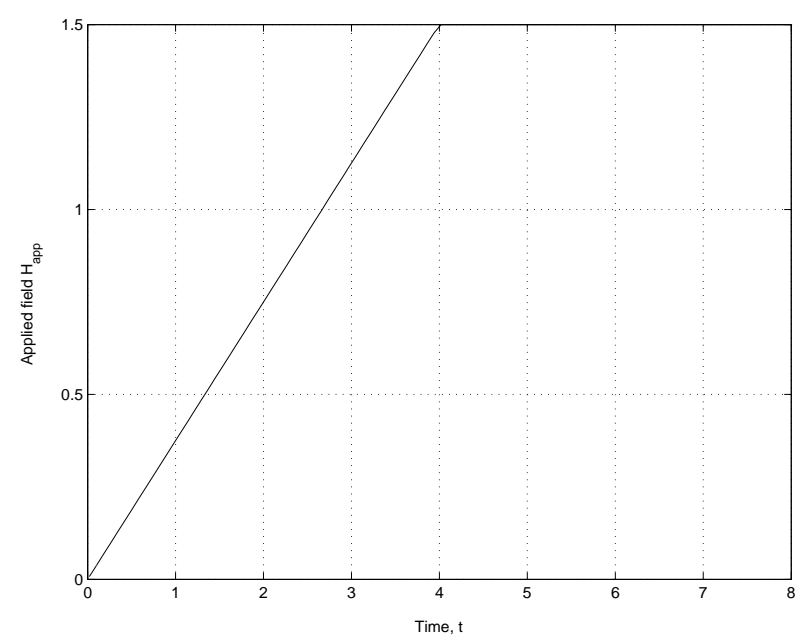

FIG. 5.7. Variation of the applied magnetic field with time for the simulations shown in figures 5.8 and 5.9 .

\begin{tabular}{|cl|}
\hline$\lambda$ & penetration depth \\
$\xi$ & coherence length \\
$d$ & typical film thickness \\
$L$ & typical film width \\
$\nu$ & vortex separation \\
$\varepsilon$ & lengthscale of pinning potential \\
$\lambda_{\text {eff }}=\lambda^{2} / d$ & effective penetration depth \\
\hline \multirow{2}{*}{ Definition of the lengthscales in Figure 6.1.}
\end{tabular}

the increased Ginzburg-Landau parameter $\kappa_{\text {eff }}=\Lambda / \Xi=\lambda \kappa / d \rightarrow \infty$, in contrast to bulk superconductors, where it corresponds to the limit $\kappa \rightarrow \infty$. This explains why thin films of even type-I superconductors will exhibit vortex solutions when the thickness becomes smaller than the penetration depth.

Following on from the London limit we considered the situation in which the vortex separation tends to zero and the vortices can be averaged to produce a vortex density.

Finally we considered critical state models, in which the pinning potential is homogenised to give stick/slip mobility laws.

In each case we found that a key parameter is $\Lambda=\lambda^{2} / L d$. If this parameter is order one, corresponding to a lateral film dimension of the same order as the effective screening length $\lambda_{\text {eff }}$, then the problems for the electric current in the film and the magnetic field outside it are coupled. If this parameter is large then the applied magnetic field passes straight through the film to leading order, and the problem for the current inside the film is decoupled. If $\Lambda$ is small, then the model also simplifies, since then the vorticity in the film determines the magntic field, which subsequently determines the current in the film.

Our hierarchy contains one or two models which have appeared elsewhere in the literature. The thin-film limit of the Ginzburg-Landau equations was considered in [8], but in the limit $d / L \rightarrow 0$ with $\xi$ and $\lambda$ fixed. Thus the model they obtained correponds to the limit $\Lambda \rightarrow \infty$.

The London model for uniform strips in the absense of vortices was considered in [16], and independently for variable thickness films in two lateral dimensions with vortices in [10,21].

The thin-film critical-state model has been considered in [29] and [31]. However, because they took the thin film limit of the bulk critical state model, in which $\lambda$ had already been set to zero, their model corresponds to the $\Lambda \rightarrow 0$ limit.

A summary of the hierarchy of models we have derived is shown in Figure 6.1, with a summary of the definitions of the various lengthscales in Table 6.1. 


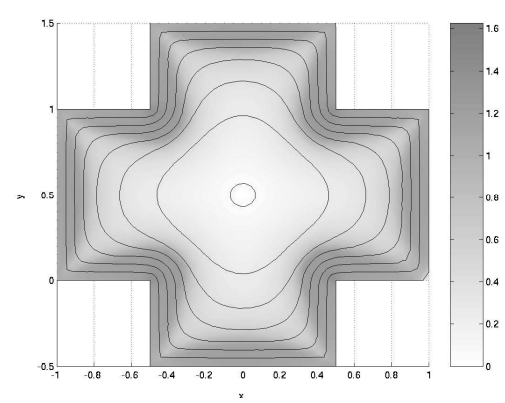

(a) $|\boldsymbol{J}|$ at time $t=1$

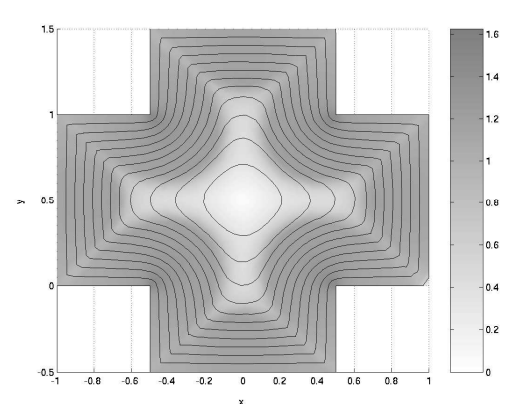

(d) $|\boldsymbol{J}|$ at time $t=2$

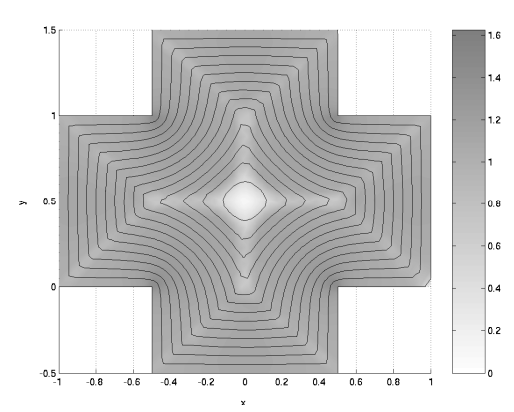

(g) $|\boldsymbol{J}|$ at time $t=3$

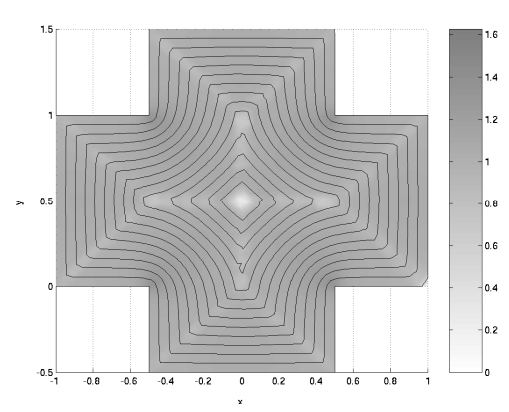

(j) $|\boldsymbol{J}|$ at time $t=4$

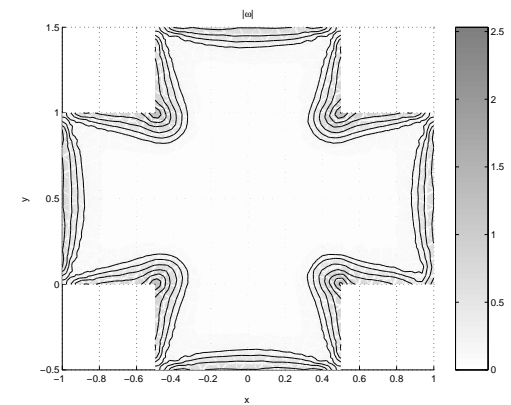

(b) $\omega$ at time $t=1$

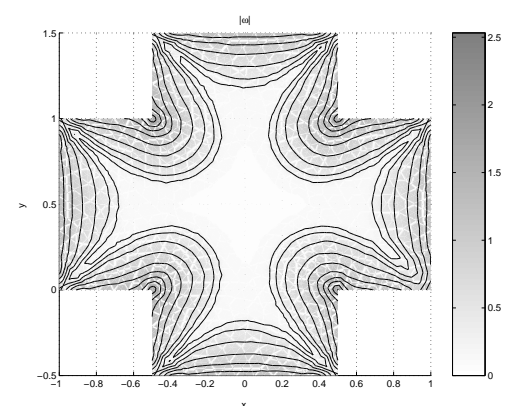

(e) $\omega$ at time $t=2$

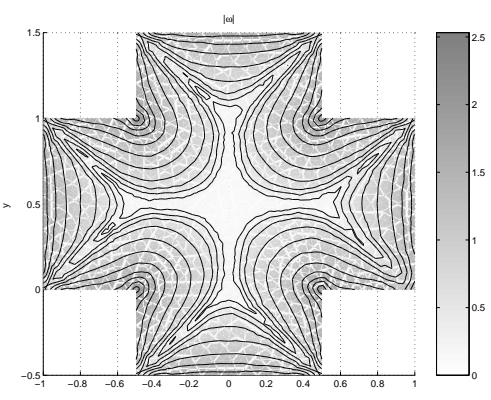

(h) $\omega$ at time $t=3$

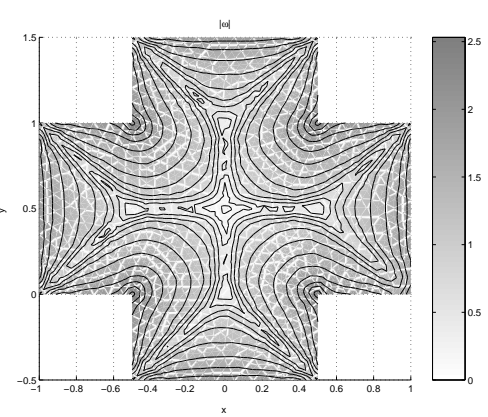

(k) $\omega$ at time $t=4$

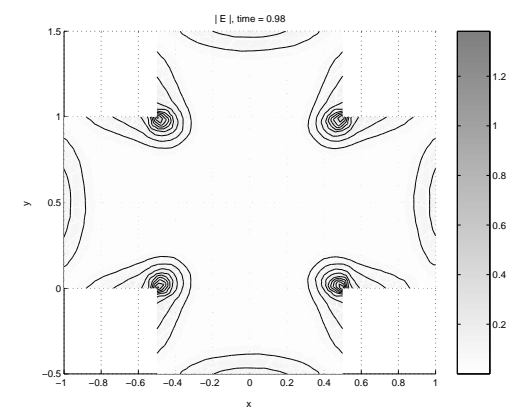

(c) $|\boldsymbol{E}|$ at time $t=1$

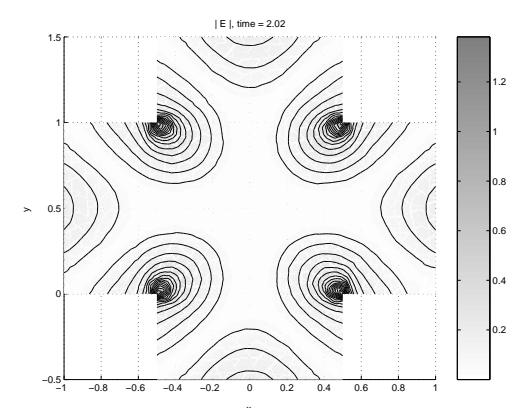

(f) $|\boldsymbol{E}|$ at time $t=2$

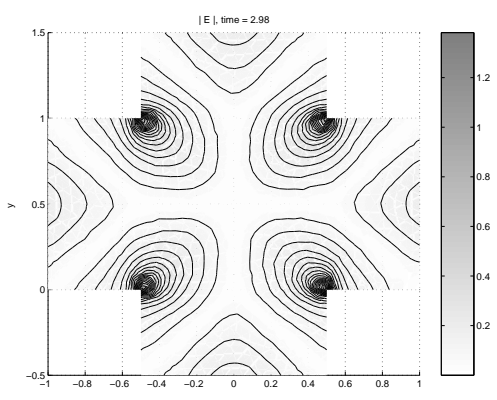

(i) $|\boldsymbol{E}|$ at time $t=3$

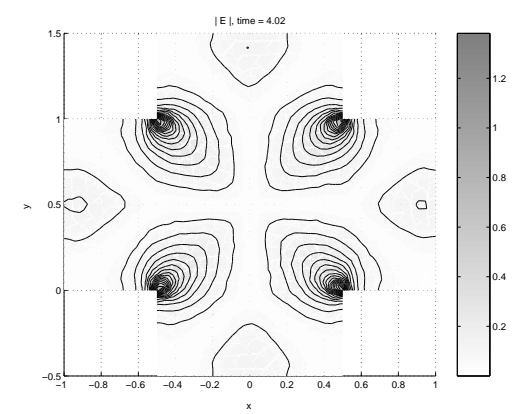

(l) $|\boldsymbol{E}|$ at time $t=4$

FIG. 5.8. Solutions of the thin-film critical-state model of a cross in an applied magnetic field in the limit $\Lambda \rightarrow 0$. The magnetic field is ramped up from zero to 1.5 over 4 time units. Numerical solution due to A. D. Grief [20] 


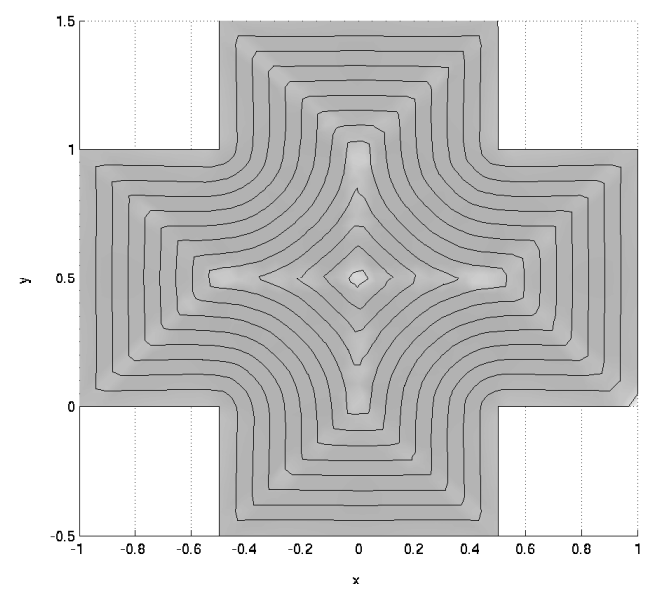

(a) $|\boldsymbol{J}|$ in steady state
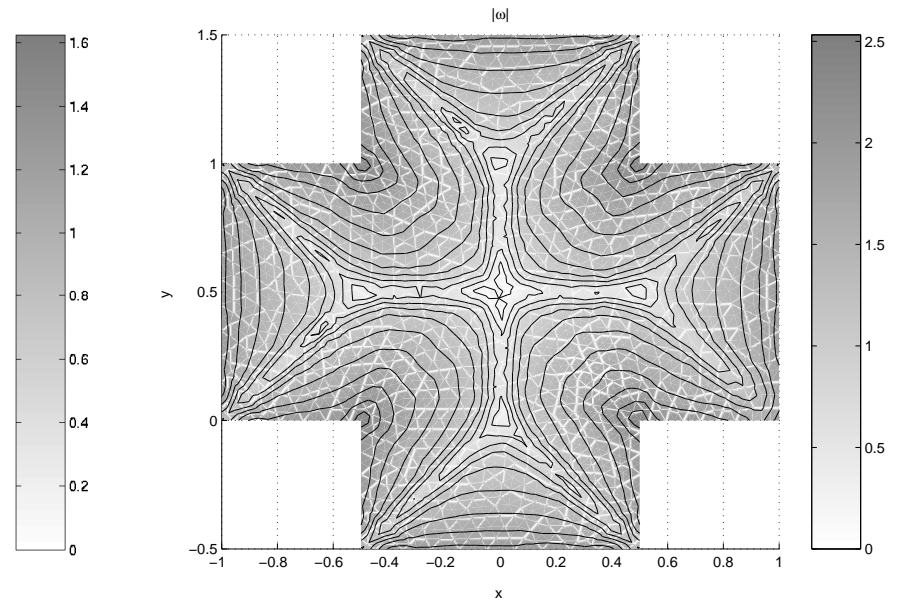

(b) $\omega$ in steady state

FIG. 5.9. Steady state of the thin-film critical-state model of a cross in an applied magnetic field of strength 1.5 in the limit $\Lambda \rightarrow 0$. Numerical solution due to A. D. Grief [20]

Acknowledgements. SJC would like to thank Andrew Grief for many useful discussions, for identifying errors in a preliminary version of this manuscript, and for kindly allowing us to present some of his numerical solutions of thin film models in figures 5.8 and 5.9.

\section{REFERENCES}

[1] I. Aranson, M. Glitterman, and B. Y. Shapiro. Onset of vortices in thin superconducting strips and wires. prb, 51(5):30923096, 1995.

[2] J. Bardeen, L. N. Cooper, and J. R. Schrieffer. Theory of superconductivity. Phys. Rev., 108:1175-1204, 1957.

[3] M. S. Berger and Y. Y. Chen. Symmetric vortices for the Ginzburg-Landau equations of superconductivity and the nonlinear desingularization phenomenon. J. Funct. Anal., 82:259-295, 1989.

[4] E. H. Brandt and M. Indenbom. Type-II superconductor strip with current in a perpendicular magnetic field. Phys. Rev. $B, 48(17): 12893-12906,1993$.

[5] S. J. Chapman. A mean-field model of superconducting vortices in three dimensions. SIAM J. Appl. Math., 55(5):1259$1274,1995$.

[6] S. J. Chapman. A hierarchy of models for type-II superconductors. SIAM Review, page to appear, 2000.

[7] S. J. Chapman, Q. Du, and M. D. Gunzburger. A Ginzburg-Landau-type model of superconducting/normal junctions including Josephson junctions. Europ. J. Appl. Math., 6:97-114, 1995.

[8] S. J. Chapman, Q. Du, and M. D. Gunzburger. A model for variable thickness superconducting thin films. ZAMP, 47(3):410-431, 1996.

[9] S. J. Chapman, A. D. Grief, S. D. Howison, M. D. McCulloch, D. Dew-Hughes, J. Moore, and C. M. Grovenor. Vortex velocity laws to I-V data for flat superconductors. IEEE Trans. Appl. Supercon., 11(1):3943-3946, 2001.

[10] S. J. Chapman and D. R. Heron. The motion of superconducting vortices in thin films of varying thickness. SIAM J. Appl. Math., 58(6):1808-1825, 1998.

[11] S. J. Chapman and G. Richardson. Motion of vortices in type-II superconductors. SIAM J. Appl. Math., 55(5):1275-1296, 1995.

[12] S. J. Chapman and G. Richardson. Vortex pinning by inhomogeneities in type-II superconductors. Physica D, 108(4):397407, 1997.

[13] X. Chen, C. M. Elliott, and Q. Tang. Shooting method for vortex solutions of a complex-valued Ginzburg-Landau equation. Proc. Roy. Soc. Edin. A, 124(6):1075-1088, 1994.

[14] J. Deang, Q. Du, M. D. Gunzburger, and J. Peterson. Vortices in superconductors: Modelling and computer simulations. Phil. Trans. A, 355(1731):1957-1968, 1997.

[15] K. Deckelnick, C. M. Elliott, and G. Richardson. Long time asymptotics for forced curvature flow with applications to the motion of a superconducting vortex. Nonlinearity, 10(3):655-678, 1997.

[16] A. T. Dorsey. Linear response of thin superconductors in perpendicular magnetic fields: An asymptotic analysis. Phys. Rev. B, 51(21):15329-15343, 1995.

[17] V. L. Ginzburg and L. D. Landau. On the theory of superconductivity. JETP, 20:1064-1082, 1950. 


\begin{tabular}{|c|c|c|}
\hline $\begin{array}{l}\text { Ginzburg-Landau model } \\
d, \lambda_{\text {eff }} \ll L \\
\xi, \nu, \varepsilon \sim L \\
\text { Sec. } 2.2 .2\end{array}$ & $\begin{array}{l}\text { Ginzburg-Landau model } \\
d \ll L \\
\xi, \lambda_{\text {eff }}, \nu, \varepsilon \sim L \\
(2.99)-(2.112)\end{array}$ & $\begin{array}{l}\text { Ginzburg-Landau model } \\
d \ll L \\
\xi, \nu, \varepsilon \sim L \\
\lambda_{\text {eff }} \gg L \\
\text { Sec. } 2.2 .2\end{array}$ \\
\hline & $\Xi \rightarrow 0$ & \\
\hline $\begin{array}{l}\text { London model } \\
\xi \ll \lambda_{\text {eff }} \ll L \\
d \ll L \\
\nu, \varepsilon \sim L \\
\text { Sec. } 3.4\end{array}$ & $\begin{array}{l}\text { London model } \\
\xi, d \ll L \\
\lambda_{\text {eff }}, \nu, \varepsilon \sim L \\
(3.41)-(3.49)\end{array}$ & $\begin{array}{l}\text { London model } \\
\xi, d \ll L \\
\nu, \varepsilon \sim L \\
\lambda_{\text {eff }} \gg L \\
\text { Sec. } 3.4\end{array}$ \\
\hline & $\nu / L \rightarrow 0$ & \\
\hline \multirow[t]{2}{*}{$\begin{array}{l}\text { Vortex-density model } \\
\xi \ll \nu \ll \lambda_{\text {eff }} \ll L \\
d \ll L \\
\varepsilon \sim L \\
(4.43)-(4.48)\end{array}$} & $\begin{array}{l}\text { Vortex-density model } \\
\xi \ll \nu \ll L \\
d \ll L \\
\lambda_{\text {eff }}, \varepsilon \sim L \\
(4.2)-(4.10),(4.13)\end{array}$ & 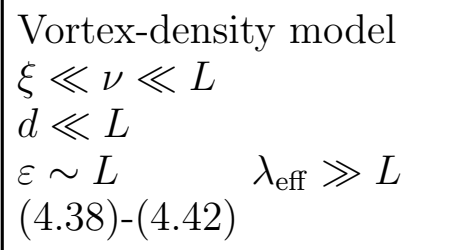 \\
\hline & $\varepsilon / L \rightarrow 0$ & \\
\hline $\begin{array}{l}\text { Critical-state model } \\
\xi \ll \nu \ll \lambda_{\text {eff }} \ll L \\
d, \varepsilon \ll L \\
(5.46)-(5.53)\end{array}$ & $\begin{array}{l}\text { Critical-state model } \\
\xi \ll \nu, \varepsilon \ll L \\
d \ll L \\
\lambda_{\text {eff }} \sim L \\
(5.7)-(5.15),(5.17)-(5.18)\end{array}$ & $\begin{array}{l}\text { Critical-state model } \\
\xi \ll \nu, \varepsilon \ll L \\
d \ll L \\
\lambda_{\text {eff }} \gg L \\
(5.30)-(5.32)\end{array}$ \\
\hline
\end{tabular}

FIG. 6.1. The hierarchy of models for superconducting thin films. Recall that $\Xi=\xi / L$ and $\Lambda=\lambda_{\text {eff }} / L$. Numbers refer to equation numbers in the text. The lengthscales are defined in Table 6.1.

[18] L. P. Gor'kov. Microscopic derivation of the Ginzburg-Landau equations in the theory of superconductivity. Soviet Phys. JETP, 9:1364-1367, 1959.

[19] L. P. Gor'kov and G. M. Éliashberg. Generalisation of the Ginzburg-Landau equations for non-stationary problems in the case of alloys with paramagnetic impurities. Soviet Phys. JETP, 27:328-334, 1968.

[20] A. D. Grief. Superconducting Thin Films. PhD thesis, University of Oxford, 2003.

[21] D. R. Heron. Mathematical Methods in Superconductivity. PhD thesis, University of Oxford, 1995.

$[22]$ A. I. Larkin and Y. N. Ovchinnikov. Influence of inhomogeneities on superconductor properties. Soviet Physics JETP, 
34(3):651-655, 1972.

[23] K. Likharev. Superconducting weak links. Rev. Modern Phys., 51:101-159, 1979.

[24] J. Pearl. Current distribution in superconducting films carrying quantized fluxiods. Appl. Phys. Lett., 5(4):65-66, 1964.

[25] L. Peres and J. Rubinstein. Vortex dynamics in U(1) Ginzburg-Landau models. Physica D, 64:299-309, 1993.

[26] G. Richardson. Instability of a superconducting line vortex. Physica D, 110(1-2):139-153, 1997.

[27] A. Schmid. A time dependent Ginzburg-Landau equation and its application to the problem of resistivity in the mixed state. Physik der Kondensierten Materie, 5:302, 1966.

[28] T. Schuster, H. Kuhn, and E. H. Brandt. Flux penetration into flat superconductors of arbitrary shape: Patterns of magnetic and electric fields and current. prb, 54(5):3514-3524, 1996.

[29] T. Schuster, H. Kuhn, E. H. Brandt, M. Indenbom, M. Koblischka, and M. Konczykowski. Flux motion in thin superconductors with inhomogeneous pinning. Phys. Rev. B, 50(22):16684, 1994

[30] M. Tinkham. Effect of fluxoid quantization on transitions of superconducting films. Phys. Rev., 129(6):2413, 1963.

[31] E. Zeldov, J. R. Clem, M. McElfresh, and M. Darwin. Magnetization and transport currents in thin superconducting films. Phys. Rev. B, 49(14):9802, 1994. 